\title{
Multilevel coarse graining and nano-pattern discovery in many particle stochastic systems
}

\author{
Evangelia Kalligiannaki ${ }^{\mathrm{a}, *}$, Markos Katsoulakis ${ }^{\mathrm{b}, \mathrm{c}}$, Petr Plecháčc ${ }^{\mathrm{a}, * *}$, Dion \\ Vlachos $^{\mathrm{d}}$ \\ ${ }^{a}$ Department of Mathematical Sciences, University of Delaware, Newark, Delaware, \\ United States \\ ${ }^{b}$ Department of Mathematics and Statistics, University of Massachusetts at Amherst, \\ United States \\ ${ }^{c}$ Department of Applied Mathematics, University of Crete and Foundation of Research \\ and Technology-Hellas, Greece \\ ${ }^{d}$ Department of Chemical Engineering, University of Delaware, Newark, Delaware, \\ United States
}

\begin{abstract}
In this work we propose a hierarchy of Monte Carlo methods for sampling equilibrium properties of stochastic lattice systems with competing short and long range interactions. Each Monte Carlo step is composed by two or more sub - steps efficiently coupling coarse and microscopic state spaces. The method can be designed to sample the exact or controlled-error approximations of the target distribution, providing information on levels of different resolutions, as well as at the microscopic level. In both strategies the method achieves significant reduction of the computational cost compared to conventional Markov Chain Monte Carlo methods. Applications in phase transition and pattern formation problems confirm the efficiency of the proposed methods.
\end{abstract}

Keywords: Markov chain Monte Carlo, coarse graining, lattice systems, phase transitions, pattern formation.

2000 MSC: 65C05, 65C20, 82B05

\footnotetext{
*Corresponding author

**Principal corresponding author

Email addresses: ekalligi@math.udel.edu (Evangelia Kalligiannaki), markos@math . umass .edu (Markos Katsoulakis), plechac@math.udel.edu (Petr Plecháč), vlachos@che.udel.edu (Dion Vlachos)
} 


\section{Introduction}

Our primary goal in this work is to develop a systematic mathematical and computational strategy for accelerating microscopic simulation methods with competing short and long range interactions, arising in numerous physical systems for instance in micromagnetics, models of epitaxial growth, etc. We propose the Multilevel Coarse Graining Monte Carlo (Multilevel CGMC) method, based on a hybrid statistical mechanics and statistics approach. The method introduces a hierarchy of Markov Chain Monte Carlo methods coupling scales and types of interactions that can sample the exact or controlled error approximations of Gibbs measures $\mu_{N, \beta}(d \sigma)=Z_{N}^{-1} e^{-\beta H_{N}(\sigma)} P_{N}(d \sigma)$ defined on a high dimensional space, $N>>1$, that can be easily generalized to any probability measure with similar properties. It is a method of constructing efficient proposal measures in Metropolis sampling using coarse-graining techniques, see also (7) below, aiming at reducing the rejection rate and the computational complexity. The key idea is a decomposition of the sampling distribution to a product measure

$$
\mu_{N, \beta}(d \sigma)=\bar{\mu}_{M, \beta}^{(0)}(d \eta) \nu_{r}(d \sigma \mid \eta),
$$

with $\eta:=\mathbf{T} \sigma$ a variable with less degrees of freedom compared to $\sigma$, de-

fined by a projection map $\mathbf{T}: \Sigma_{N} \rightarrow \bar{\Sigma}_{M}, M<N . \bar{\mu}_{M, \beta}^{(0)}(d \eta)$ is a measure with a simple explicit representation approximating the marginal $\bar{\mu}_{M, \beta}(d \eta)=$ $\mu_{N, \beta} \circ \mathbf{T}^{-1}(d \eta)$ and $\nu_{r}(d \sigma \mid \eta)$ is a uniquely defined (prior) measure, responsible for reconstructing variables $\sigma$ given $\eta$, [19]. Such a two-level measure decomposition can be trivially extended to a multi-level setting where (1) can include different resolution levels interpolating between a coarser level and the microscopic one $\sigma$.

In the following we describe a Monte Carlo step of a two-level CGMC method:

1. Sample $\eta$ from $\bar{\mu}_{M, \beta}^{(0)}(d \eta)$, using Coarse Grained (CGMC) samplers [18, 17]. Appropriate coarse grained measures have been evaluated in earlier work via cluster expansions that can be easily constructed with available analytical error estimates ensuring that such approximations are controllable [20].

2. Conditioned on such $\eta$, obtained in Step 1, we sample $\nu_{r}(d \sigma \mid \eta)$ via an Accept/Reject method. 
A schematic description of this procedure can be seen in Figure 1. Better proposals constructed in Step 1 will lead to fewer rejections in Step 2, furthermore, there is no need to consider all possible microscopic proposals since at the coarse step we do a first screening. Instead of further approximating the sampling measure, as is done with cluster expanding (5) keeping the typically computationally expensive multi-body higher order terms [23], we use the hybrid statistical and statistics approach that construct $\nu_{r}(d \sigma \mid \eta)$. Even when CGMC provide less accurate approximations, the multilevel CGMC approach can refine the results by the Accept/Reject step in the finer space. A key issue is how to sample conditionally from $\nu_{r}$ in an efficient manner in Step 2, picking an appropriate coarse proposal in Step 1.

The necessary ingredient for the applicability of the method is a decomposition of the form (1), which includes a possibly less accurate coarse-grained measure and the correcting accept/reject Step 2 above. This formulation can make the proposed method extentable to off-lattice systems where various coarse-graining schemes are already available [26, 12, 2], although without controlled-error approximations. In such off-lattice systems we typically have two main features: a presence of short and longer interactions, as well as comparable energy and entropy, hence fluctuations are expected to be important in the modelling and simulation.

Systems with smooth long or intermediate range interactions are well approximated by coarse graining techniques [17, 19, 24], and CGMC are reliable simulation methods with controlled-error approximations, both for observables and loss of information [21, 20]. Furthermore, models where only short-range interactions appear are inexpensive to simulate with conventional methods. However, when both short and long range interactions are present, the conventional methods become prohibitively expensive, and CG error estimates are not applicable. The proposed method can handle such systems efficiently by either compressing only the long range interactions, and sample with CGMC with low computational cost, at Step 1 and include the short range part at the accept/reject Step 2, or compress all types of interactions for Step 1, and correct appropriately in Step 2.

A wide literature exists on sophisticated Markov Chain Monte Carlo (MCMC) methods designed to accelerate simulations for large systems, applying for example parallelising techniques and/or constructing good first approximations (proposals) in Metropolis sampling [9], [31]. In [10] Efendiev et.al., propose the Preconditioning MCMC, a two stage Metropolis method, applied to inverse problems of subsurface characterization. Our algorithm 
shares the same idea of constructing a proposal density based on meso /macro-scopic properties of the model studied and taking advantage of the first stage rejections. Several methods where the trial density is built up sequentially with stage-wise rejection decision appear, [3], [28]. There are also some similarities with simulated sintering, and transdimensional MCMC, see [29, 28] and references therein. However, the novelty of our method lies on the construction of the variable dimensionality (and level of coarse-graining) state spaces and the corresponding Gibbs measures relies on statistical mechanics tools that allow a systematic control of the error from one level of coarse-graining to the next. The interplay of different levels of compressed spaces appears also in spatial multi-grid methods coupled with CGMC sampling, studied in [6] [4], for accelerating lattice kinetic Monte Carlo simulations where however the proposed methods are not exact and do not necessarily provide controlled-error approximations. Various attempts appear on parallelising Monte Carlo simulations, based on a parallel resolution, such as parallel kinetic CGMC [1] and a combination of CGMC and parallel tempering [35]. In a follow up work we extend our framework for multilevel CGMC to accelerate sampling of evolution processes on large lattice systems [14]. There we develop multilevel Kinetic Monte Carlo algorithms, based on the known from coarse graining techniques different level approximating processes.

In Section 2 we introduce microscopic lattice systems and provide a brief review of coarse graining methods. We also provide the Metropolis-Hastings MCMC method for numerical simulations and describe microscopic processes. We introduce the multilevel CGMC (ML CGMC) method in Section 3 and provide the mathematical analysis that ensures the theoretical validity of the method. Section 4 provides a full comparison of the computational complexity between the classical and the multilevel Metropolis introduced here. More specifically, in Theorem 2 we prove comparative estimates on their mixing times via spectral gap estimates. In turn such spectral estimates are obtained through suitable relative bounds on their respective Dirichlet forms. Concluding, our analysis shows that ML CGMC has substantial savings over the classical MH algorithm, generating cheaply proposals with small or controllable rejection rates. Sections 5 and 6 give example applications of the multilevel method in canonical and microcanonical sampling. In Section 5.1 a benchmark example is employed in order to demonstrate an explicit application of Theorem 2. In subsection 5.2 an order one improvement of the coarse graining error in a phase transition regime is achieved when applying 
the multilevel CGMC method with a potential splitting approach, for a Kac type potential with algebraic decay. Finally in Section 6 we study nanopattern formation in surface diffusion induced by a Morse type potential, and verify that the proposed method can provide correctly microscopic details.

\section{Stochastic lattice systems at equilibrium}

We consider an Ising-type system on a periodic $d$-dimensional lattice $\Lambda_{N}$ with $N=n^{d}$ lattice sites. At each site $x \in \Lambda_{N}$ we define a spin variable $\sigma(x)$ taking values in a finite set. For instance, in a lattice gas model $\sigma(x) \in\{0,1\}$ describes that the site $x$ is vacant or occupied by an atom. The state of the system is described by a configuration $\sigma \in \Sigma_{N}=\{0,1\}^{\Lambda_{N}}$. The interaction energy of the system, e.g., interacting particles in the lattice gas model, is defined by the Hamiltonian $H_{N}$. We assume systems where the particles interact only through a pair-wise potential and thus the Hamiltonian takes the form $\sigma=\left\{\sigma(x): x \in \Lambda_{N}\right\}$

$$
H_{N}(\sigma)=-\frac{1}{2} \sum_{x \in \Lambda_{N}} \sum_{y \neq x} J(x-y) \sigma(x) \sigma(y)+\sum_{x \in \Lambda_{N}} h(x) \sigma(x),
$$

where $h$ is an external field. Equilibrium states at the inverse temperature $\beta$ are described by the (canonical) Gibbs probability measure on the space $\Sigma_{N}$

$$
\mu_{N, \beta}(d \sigma)=Z_{N}^{-1} e^{-\beta H_{N}(\sigma)} P_{N}(d \sigma),
$$

where $Z_{N}$ is the normalizing constant (partition function). Furthermore, the product Bernoulli distribution $P_{N}(d \sigma)$ is the prior distribution on $\Lambda_{N}$ representing distribution of states in a non-interacting system, or equivalently at $\beta=0$, when thermal fluctuations-disorder-associated with the product structure of $P_{N}(d \sigma)$ dominates. By contrast at zero temperature, $\beta=\infty$, interactions and hence order, prevail. Finite temperatures, $0<\beta<\infty$, describe intermediate states, including possible phase transitions between ordered and disordered states.

The coarse-graining techniques have been developed in order to study the behaviour in the regimes when the size of the system $N \rightarrow \infty$. In the series of papers [17, 18, 25] the authors initiated the development of coarse-graining (CG) as a computational tool for accelerating Monte Carlo simulations of stochastic lattice dynamics. The coarse-grained model is constructed on a coarse grid $\bar{\Lambda}_{M}$ by dividing $\Lambda_{N}$ into $M$ coarse cells, each of which contains 
$Q$ (micro-)cells, typically $Q=q^{d}$ with the coarse-graining scale $q$ in each dimension. Each coarse cell is denoted by $C_{k}, k \in \bar{\Lambda}_{M}$. A typical choice for the coarse variable in the context of Ising-type models is the block-spin over each coarse cell $C_{k}$,

$$
\eta:=\left\{\eta(k)=\sum_{x \in C_{k}} \sigma(x): k \in \bar{\Lambda}_{M}\right\},
$$

defining the coarse graining map $\mathbf{T}: \Sigma_{N} \rightarrow \bar{\Sigma}_{M}, \mathbf{T} \sigma=\eta$. The exact coarse-grained Gibbs measure is given (with a slight abuse of notation) by $\bar{\mu}_{M, \beta}=\mu_{N, \beta} \circ \mathbf{T}^{-1}$, written in a more convenient form

$$
\bar{\mu}_{M, \beta}(d \eta)=\frac{1}{\bar{Z}_{M}} e^{-\beta \bar{H}_{M}(\eta)} \bar{P}_{M}(d \eta),
$$

where $\bar{P}_{M}(d \eta)=P_{N} \circ \mathbf{T}^{-1}$ defines the coarse-grained prior measure. The exact coarse-grained Hamiltonian is defined by the renormalization group map, see, e.g., [11],

$$
e^{-\beta \bar{H}_{M}(\eta)}=\mathbb{E}\left[e^{-\beta H_{N}} \mid \eta\right]=\int e^{-\beta H_{N}(\sigma)} P_{N}(d \sigma \mid \eta) .
$$

The conditional prior $P_{N}(d \sigma \mid \eta)$ is the probability of having a microscopic configuration $\sigma$, given a coarse configuration $\eta$.

Although typically $\bar{P}_{M}(d \eta)$ is easy to calculate the exact computation of the coarse-grained Hamiltonian $\bar{H}_{M}(\eta)$ given by (5) is, in general, impossible even for moderately small values of $N$. Therefore suitable approximations have to be constructed. An initial approximation can be then proposed by the technique of cluster expansions, [34] providing improved approximation at suitable phase regimes. The corresponding first order CG Hamiltonian is explicitly given, [20],

$$
\begin{aligned}
\bar{H}^{(0)}(\eta)= & -\frac{1}{2} \sum_{k \in \bar{\Lambda}_{M}} \sum_{l \neq k} \bar{J}(k, l) \eta(k) \eta(l)-\frac{1}{2} \bar{J}(0,0) \sum_{k \in \bar{\Lambda}_{M}} \eta(k)(\eta(k)-1) \\
& +\sum_{k \in \bar{\Lambda}_{M}} \bar{h} \eta(k),
\end{aligned}
$$

where the coarse-grained interactions are evaluated explicitly by averaging over the cells $k, l \in \bar{\Lambda}_{M}$

$$
\bar{J}(k, l)=\frac{1}{q^{2}} \sum_{x \in C_{k}} \sum_{y \in C_{l}} J(x-y), \bar{J}(k, k)=\frac{1}{q(q-1)} \sum_{x \in C_{k}} \sum_{y \in C_{k}, y \neq x} J(x-y),
$$


defining the coarse grained Gibbs measure

$$
\bar{\mu}_{M, \beta}^{(0)}(d \eta)=\frac{1}{\bar{Z}^{(0)}} e^{-\beta \bar{H}^{(0)}(\eta)} \bar{P}_{M}(d \eta) .
$$

The coarse-graining of systems with purely long- or intermediate-range interactions was studied using cluster expansions in [20, 2, 19, 22]. In many applications the long-range potentials exhibit scaling property

$$
J(x-y)=L^{-d} V\left(\frac{n}{L}|x-y|\right), x, y \in \Lambda_{N},
$$

where $V \in C^{1}([0, \infty))$ and it is normalized to ensure that the strength of the potential $J$ is essentially independent of $L$, i.e., $\sum_{x \neq 0} J(x) \sim \int_{0}^{\infty} V(r) d r$. The constant $L$ can be interpreted as a (characteristic) interaction range of the potential. For example, if we have $V$ with properties $V(r)=V(-r)$, $V(r)=0,|r|>1$, then a spin at the site $x$ interacts with its neighbours which are at most $L$ lattice points away from $x$. One of the results therein is on deriving error estimates in terms of the specific relative entropy $\mathcal{R}(\mu \mid \nu):=N^{-1} \sum_{\sigma} \log \{\mu(\sigma) / \nu(\sigma)\} \mu(\sigma) \quad$ between the corresponding equilibrium Gibbs measures. Note that the scaling factor $N^{-1}$ is related to the extensivity of the system, hence the proper error quantity that needs to be tracked is the loss of information per particle.

$$
\mathcal{R}\left(\bar{\mu}_{M, \beta}^{(0)} \mid \mu_{N, \beta} \circ \mathbf{T}^{-1}\right)=\mathcal{O}\left(\epsilon^{2}\right), \quad \epsilon \equiv \beta\|\nabla V\|_{1}\left(\frac{q}{L}\right),
$$

Systems with short and long range interactions. One of our goals in this work is to study systems where in addition to the long-range potential we have a short range

$$
K(x-y)=S^{-d} V_{s}\left(\frac{n}{S}|x-y|\right), x, y \in \Lambda_{N},
$$

with $V_{s}$ having similar properties as $V$ in (8) and $S \ll L$ distinguishing the short and long range nature of interactions. A typical case of a shortrange potential is encountered in the nearest-neighbour Ising model where $K(x-y)=K=$ constant, for $|x-y|=S=1$ and zero otherwise. The new Hamiltonian including the contributing energy from the short range potential $H_{s}(\sigma)$ and the long range part $H_{l}(\sigma)$ is

$$
H_{N}(\sigma)=H_{s}(\sigma)+H_{l}(\sigma) .
$$


Study of equilibrium properties of stochastic lattice systems mainly accounts for the evaluation of averages over the coarse-grained $\bar{\mu}_{M, \beta}(d \eta)$ or the microscopic $\mu_{N, \beta}(d \sigma)$ Gibbs measures of observables $\phi: \Sigma_{N} \rightarrow \mathbb{R}$, i.e.,

$$
\mathbb{E}[\phi]=\int_{\Sigma_{N}} \phi(\sigma) \mu_{N, \beta}(d \sigma) .
$$

Numerical methods evaluating equilibrium averages are the Markov Chain Monte Carlo (MCMC) methods, among which the most widely used is the Metropolis - Hastings (MH) method [30, 13]. Metropolis-Hastings algorithm generates proposals $\sigma^{\prime}$, for the evolution from the configuration $\sigma$ to $\sigma^{\prime}$, that are defined by the proposal probability transition kernel $\rho\left(\sigma^{\prime}, \sigma\right)$. The proposal $\sigma^{\prime}$ is accepted with probability $\alpha\left(\sigma, \sigma^{\prime}\right)$ or rejected with the probability $1-\alpha\left(\sigma, \sigma^{\prime}\right)$. Let $X_{0}=\sigma_{0}$ be an arbitrary initial configuration, the $n$-th iteration of the algorithm consists of the following steps

\section{Algorithm 1 (Metropolis-Hastings algorithm). Given $X_{n}=\sigma$}

Step 1 Generate $X_{n}^{\prime}=\sigma^{\prime} \sim \rho\left(\sigma^{\prime}, \sigma\right)$.

Step 2 Accept-Reject

$$
X_{n+1}= \begin{cases}X_{n}^{\prime}=\sigma^{\prime} & \text { with probability } \alpha\left(\sigma, \sigma^{\prime}\right), \\ X_{n}=\sigma & \text { with probability } 1-\alpha\left(\sigma, \sigma^{\prime}\right),\end{cases}
$$

with the acceptance probability depending on the energy difference between configurations $\sigma$ and $\sigma^{\prime}, \Delta H_{N}\left(\sigma, \sigma^{\prime}\right)=H_{N}\left(\sigma^{\prime}\right)-H_{N}(\sigma)$,

$$
\alpha\left(\sigma, \sigma^{\prime}\right)=\min \left\{1, \exp \left\{-\beta \Delta H_{N}\left(\sigma, \sigma^{\prime}\right)\right\} \frac{\rho\left(\sigma^{\prime}, \sigma\right)}{\rho\left(\sigma, \sigma^{\prime}\right)}\right\} .
$$

The algorithm generates an ergodic Markov chain $\left\{X_{n}\right\}$ in the state space $\Sigma_{N}$, with the stationary measure $\mu_{N, \beta}(d \sigma)$. Ergodicity ensures the convergence of empirical averages $\frac{1}{n} \sum_{i=1}^{n} \phi\left(X_{i}\right)$ to the desired mean $\mathbb{E}[\phi]$, for any $\phi \in$ $L^{1}\left(\mu_{N, \beta}\right)$. It is easy to deduce the probability transition kernel associated to $\mathrm{MH}$ Algorithm 1

$$
\mathcal{K}_{c}\left(\sigma, \sigma^{\prime}\right)=\alpha\left(\sigma, \sigma^{\prime}\right) \rho\left(\sigma, \sigma^{\prime}\right)+\left[1-\int_{\Sigma} \alpha\left(\sigma, \sigma^{\prime}\right) \rho\left(\sigma, \sigma^{\prime}\right) d \sigma^{\prime}\right] \delta\left(\sigma^{\prime}-\sigma\right) .
$$

where $\delta$ denotes the Dirac measure. 
Depending on whether one considers microcanonical or canonical ensemble the ergodic Markov chain $\left\{X_{n}\right\}$ can be defined by spin-exchange dynamics that preserve the order parameter or spin-flip dynamics respectively [27], [7]. In the spin-exchange the proposed new configuration $\sigma^{\prime}=\sigma^{(x, y)}$ is obtained from $\sigma$ by interchanging the spins at $x$ and $y$, for nearest-neighbour sites $x$ and $y$.

$$
\sigma^{(x, y)}(z)= \begin{cases}\sigma(y), & \text { when } z=x \\ \sigma(x), & \text { when } z=y \\ \sigma(z), & \text { otherwise. }\end{cases}
$$

Analogously for the spin-flip $\sigma^{\prime}=\sigma^{(x)}$ is obtained from $\sigma$ by flipping the spin value at site $x$

$$
\sigma^{(x)}(z)= \begin{cases}1-\sigma(x), & \text { when } z=x \\ \sigma(z), & \text { otherwise }\end{cases}
$$

\section{The multilevel CGMC Metropolis-Hastings method}

We present in detail and generalize the Coupled Metropolis-Hastings method originally proposed in [15]. We introduce a multi-level MetropolisHastings method, where each level corresponds to a configuration space resolution level, and provide the associated mathematical analysis that ensures the theoretical validity of the method.

Let $\left\{\bar{\Sigma}_{M_{j}}\right\}_{j=0}^{I}$ denote a hierarchy of coarse spaces derived from the microscopic space $\Sigma_{N}=: \bar{\Sigma}_{M_{0}}$ by a family of mappings $\mathbf{T}_{j}: \bar{\Sigma}_{M_{j}} \rightarrow \bar{\Sigma}_{M_{j+1}}$, $\mathbf{T}_{j} \eta_{j}=\eta_{j+1}$, for $N=M_{0}>M_{1}>\cdots>M_{I}$. The variables $\eta_{j}$ denote configurations on spaces $\bar{\Sigma}_{M_{j}}$, with $\eta_{0}=: \sigma$ referring to the microscopic variable on $\Sigma_{N}$. The method is composed by a sequence of $I+1$ Metropolis-Hastings steps each one designed to generate samples from $\bar{\Sigma}_{M_{j}}$ given a coarser sample from $\bar{\Sigma}_{M_{j+1}}$. Properly constructed measures on $\bar{\Sigma}_{M_{j}}, j>0$ form the basis for constructing efficient proposal kernels for Metropolis-Hastings algorithm allowing sampling of large systems. The interplay between different resolution spaces is controlled by the hierarchy of projection mappings $\mathbf{T}_{j}$ and corresponding inverse mapping procedures (reconstruction). Appropriate reconstruction measures $\nu_{r, j}\left(d \eta_{j} \mid \eta_{j+1}\right)$ are constructed in view of decompositions similar to (13), for the each pair of Gibbs measures $\mu_{j}\left(d \eta_{j}\right)$ and $\mu_{j+1}\left(d \eta_{j+1}\right)$, see Figure 1, For the sake of exposition we describe in detail the two-level $C G M C$ method, in which two different scale state spaces $\bar{\Sigma}_{M_{j_{1}}}=\Sigma_{N}$ and $\bar{\Sigma}_{M_{j_{2}}}=\Sigma_{M}, M<N$ are involved. 


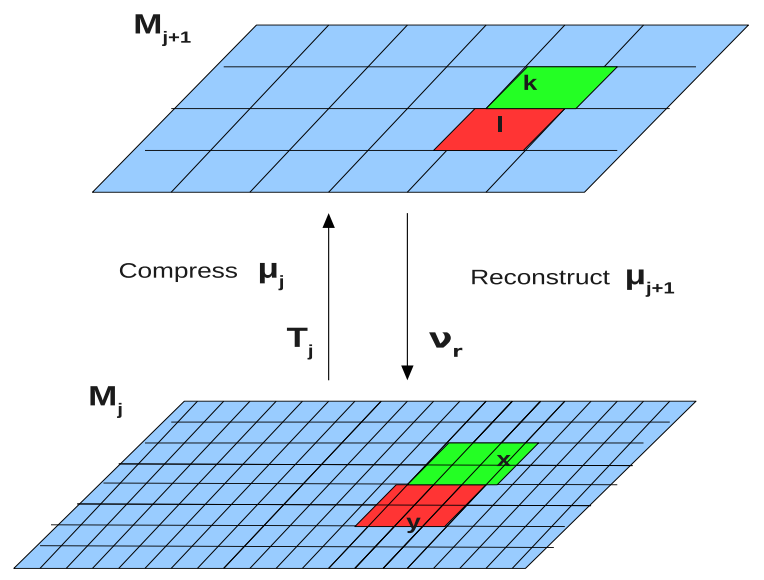

Figure 1: Schematic of a two-level ML CGMC. Information exchange between coarser and finer resolutions. Step 1: Sample $\mu_{j+1}\left(d \eta_{j+1}\right)$, Step 2: Reconstruct with $\nu_{r}\left(d \eta_{j} \mid \eta_{j+1}\right)$ such that $\mu_{j}\left(d \eta_{j}\right)=\mu_{j+1}\left(d \eta_{j+1}\right) \nu_{r}\left(d \eta_{j} \mid \eta_{j+1}\right)$.

The ML CGMC method is a hybrid statistical mechanics and statistics approach based on a product measure decomposition of the target distribution

$$
\mu_{N, \beta}(d \sigma)=\bar{\mu}_{M, \beta}^{(0)}(d \eta) \nu_{r}(d \sigma \mid \eta) .
$$

The principal idea is that computationally inexpensive CG simulations will reproduce the large scale structure and subsequently microscopic information will be added through the microscopic reconstruction. The two levels of the method are described by:

1. Marginal approximation and CGMC sampling. $\bar{\mu}_{M, \beta}^{(0)}(d \eta)$, given in (7), an approximation of marginal $\bar{\mu}_{M, \beta}=\mu_{N, \beta} \circ \mathbf{T}^{-1}$, is described explicitly with a controllable error [20].

2. Microscopic reconstruction. Conditioned on $\eta$, obtained by sampling from $\bar{\mu}_{M, \beta}^{(0)}(d \eta)$, we sample $\nu_{r}(d \sigma \mid \eta)$ via a simple inverse-mapping distribution $\mu_{r}(d \sigma \mid \eta)$ and an Accept/Reject method. Measure $\nu_{r}(d \sigma \mid \eta)$ is responsible for the reconstruction procedure of the fine configuration $\sigma$ 
constrained on the coarse configuration $\eta$.

More specifically, reconstruction is the reverse procedure to coarse-graining, i.e., reproducing microscopic properties directly from CG simulations. A detailed discussion on reconstruction can be found in [19, 15]. Note that $\nu_{r}(d \sigma \mid \eta)$ is a finite measure, uniquely defined by (13) given $\mu_{N, \beta}(d \sigma)$ and $\bar{\mu}_{M, \beta}^{(0)}(d \eta)$,

In view of Decomposition (13) we propose the two-level CGMC algorithm composed of two Metropolis-Hastings steps. The first step samples the measure $\bar{\mu}_{M, \beta}^{(0)}(d \eta)(7)$, on the coarse space $\bar{\Sigma}_{M}$, using an arbitrary proposal transition kernel $\bar{\rho}\left(\eta, \eta^{\prime}\right)$ to produce coarse trial samples $\eta^{\prime}$. The second step, performed only if the coarse trial sample is accepted, consists of the simple reconstruction from the coarse state $\eta^{\prime}$ with $\mu_{r}(d \sigma \mid \eta)$ and an accept/reject method. If a trial coarse sample is rejected the second step is not performed and no computational time is wasted on checking fine trial samples that are most likely to be rejected. The two-level CGMC algorithm is defined as follows: Let $Y_{0}=\sigma_{0}$ be an arbitrary initial configuration, for $n=0,1,2, \ldots$,

\section{Algorithm 2 (Two-level CGMC MH Algorithm). Given $Y_{n}=\sigma$}

Step 1 Compute the coarse configuration $\eta=\mathbf{T} \sigma$.

Step 2 Generate a coarse sample $\eta^{\prime} \sim \bar{\rho}\left(\eta, \eta^{\prime}\right)$.

Step 3 Coarse Level Accept-Reject.

Accept $\eta^{\prime}$ with probability:

$$
\alpha_{C G}\left(\eta, \eta^{\prime}\right)=\min \left\{1, e^{-\beta \Delta \bar{H}^{(0)}\left(\eta, \eta^{\prime}\right)} \frac{\bar{\rho}\left(\eta^{\prime}, \eta\right)}{\bar{\rho}\left(\eta, \eta^{\prime}\right)}\right\}
$$

if $\eta^{\prime}$ is accepted then proceed to Step 4,

else generate a new coarse sample Step 2.

Step 4 Reconstruct $\sigma^{\prime}$ given the coarse trial $\eta^{\prime}$,

$$
\sigma^{\prime} \sim \mu_{r}\left(\cdot \mid \eta^{\prime}\right)
$$

Step 5 Fine Level Accept-Reject.

Accept $\sigma^{\prime}$ with probability

$$
\alpha_{f}\left(\sigma, \sigma^{\prime}\right)=\min \left\{1, e^{-\beta\left[\Delta H_{N}\left(\sigma, \sigma^{\prime}\right)-\Delta \bar{H}^{(0)}\left(\eta, \eta^{\prime}\right)\right]} \frac{\mu_{r}(\sigma \mid \eta)}{\mu_{r}\left(\sigma^{\prime} \mid \eta^{\prime}\right)}\right\} .
$$


where $\Delta H_{N}\left(\sigma, \sigma^{\prime}\right)=H_{N}\left(\sigma^{\prime}\right)-H_{N}(\sigma)$, and $\Delta \bar{H}^{(0)}\left(\eta, \eta^{\prime}\right)=\bar{H}^{(0)}\left(\eta^{\prime}\right)-\bar{H}^{(0)}(\eta)$.

In terms of Metropolis-Hastings Algorithm 1, the method is generating trial samples $\sigma^{\prime}$ from proposal kernel $\rho\left(\sigma, \sigma^{\prime}\right)$, with stationary measure

$\bar{\mu}_{M, \beta}^{(0)}(d \eta) \mu_{r}(d \sigma \mid \eta)$, that depends on the statistical mechanics properties of the system. This fact leads to an increase of the acceptance rate of the $\mathrm{MH}$ method, see for example Figure 4a, Indeed, consider the canonical ensemble for $x \in \Lambda_{N}$ such that $x \in C_{k}, k \in \bar{\Lambda}_{M}$ with a potential $J(x-y)$ (8), where the following estimate holds [18],

$$
\Delta H_{N}\left(\sigma, \sigma^{(x)}\right)-\Delta \bar{H}^{(0)}\left(\eta, \eta^{(k)}\right)=\mathcal{O}\left(Q /(2 L+1)^{d}\right)
$$

with $\eta^{(k)}=\mathbf{T} \sigma^{(x)}$. This estimate shows that the second level acceptance probability $\alpha_{f}\left(\sigma, \sigma^{\prime}\right)$ is controlled by the coarsening parameter, i.e. it is close to 1 for $Q /(2 L+1)^{d}<<1$ and most of the coarse samples entering the second level will be accepted. Thus, for fixed $L$, varying the coarsening parameter we can control the effectiveness of the method as a balance between acceptance rate and computational cost. That is between small values of $Q$, leading to high acceptance rate, and larger $Q$, leading to lower computational cost at sampling at the coarse space. We elaborate with this issue in detail in Section 4.

\subsection{Examples of multi-level decompositions}

In this section we elaborate on how to select the prior measure $\nu_{r}(d \sigma \mid \eta)$, that furthermore determines how to perform a multi-level decomposition of the Gibbs measure. The selection of $\nu_{r}(d \sigma \mid \eta)$ depends on two features (a) the choice of the coarse grained measure $\bar{\mu}_{M, \beta}^{(0)}(d \eta)$, that is whether we compress the entire interaction potential or split to a short and long range part and (b) on sampling the exact or an approximation of the microscopic measure $\mu_{N, \beta}(d \sigma)$.

Corrections. With this strategy all types of interactions are compressed and incorporated into the first level of sampling with $\bar{H}^{(0)}(\eta)$, as is depicted in the algorithmic description of the method Algorithm 2. The reconstructive and corrective characteristics of $\nu_{r}(d \sigma \mid \eta)$ appear explicitly in the following formulation, that is reconstruct with the uniform conditional distribution $\mu_{r}(d \sigma \mid \eta)=P_{N}(d \sigma \mid \eta)$ and correct with $H_{N}(\sigma)-\bar{H}^{(0)}(\eta)$,

$$
\nu_{r}(d \sigma \mid \eta)=\bar{Z}_{M}^{(0)} Z_{N}^{-1} \exp \left\{-\beta\left[H_{N}(\sigma)-\bar{H}^{(0)}(\eta)\right]\right\} P_{N}(d \sigma \mid \eta) .
$$


Potential splitting. An alternative approach is considered based on splitting the inter particle interactions into short- and long-range terms. A decomposition of the coarse-graining of the interaction potential can be justified and optimized by known error estimates, see [2]. These estimates suggest a natural way to split the potential into a short-range piece $K(x-y)$ with possible singularities and a locally integrable (or smooth) long-range decaying component $J(x-y)$.

Here we suggest to sample on the coarse step according to the effective Hamiltonian $\bar{H}_{l}^{(0)}(\eta)$, corresponding only to the long-range depended energy $H_{l}(\sigma)$, as in (11), that suggests a rearrangement of decomposition (13) where

$$
\nu_{r}(d \sigma \mid \eta)=\bar{Z}_{l M}^{(0)} Z_{N}^{-1} \exp \left\{-\beta\left[H_{s}(\sigma)+H_{l}(\sigma)-\bar{H}_{l}^{(0)}(\eta)\right]\right\} P_{N}(d \sigma \mid \eta) .
$$

In the two-level CGMC sampling the costly long-range part is involved only in the coarse updating where the number of operations to calculate energy differences is reduced and coarsening of the short-range potential is avoided [23].

Approximate $C G$. In many applications where meso/macroscopic information is sufficient CGMC sampling is reliable for long-range potentials and the error when neglecting terms $\Delta H_{l}\left(\sigma, \sigma^{\prime}\right)-\Delta \bar{H}_{l}^{(0)}\left(\eta, \eta^{\prime}\right)$ is small. In this strategy we suggest to neglect these terms, despite the encountering of the approximating error, benefiting from a further reduction of the computational complexity, see Section 4. As a result Algorithm 2 is sampling from a probability measure approximating $\mu_{N, \beta}(d \sigma)$,

$$
\mu_{N, \beta}^{(0)}(d \sigma) \propto \exp \left\{-\beta H_{s}(\sigma)-\beta H_{l}^{(0)}(\eta)\right\} P_{N}(d \sigma \mid \eta) \bar{\mu}_{M, \beta}^{(0)}(d \eta) .
$$

\subsection{Reversibility of the Multilevel $C G M C$}

Mathematical analysis for the two-level CGMC method is studied in this section for a broad class of probability measures. The method can be generalized to the sampling of any probability measure $\mu(d \sigma)$ on a countable configuration space $\Sigma$, by coupling properly configurations between a hierarchy of coarser and finer spaces. Since almost any probability density $\mu(\sigma)$ can be written in the form $e^{-H(\sigma)}$ the method can applied to any model for which one can properly define a function $H(\sigma)$ and the hierarchy of coarse spaces and densities. The following arguments are straightforward but necessary to prove that the algorithm samples processes with the desired stationary measure. Algorithm 2 is defined by the acceptance probabilities

$$
\alpha_{C G}\left(\eta, \eta^{\prime}\right)=\min \left\{1, \frac{\bar{\mu}^{(0)}\left(\eta^{\prime}\right) \bar{\rho}\left(\eta^{\prime}, \eta\right)}{\bar{\mu}^{(0)}(\eta) \bar{\rho}\left(\eta, \eta^{\prime}\right)}\right\},
$$


and

$$
\alpha_{f}\left(\sigma, \sigma^{\prime}\right)=\min \left\{1, \frac{\mu\left(\sigma^{\prime}\right) \bar{\mu}^{(0)}(\eta) \mu_{r}(\sigma \mid \eta)}{\mu(\sigma) \bar{\mu}^{(0)}\left(\eta^{\prime}\right) \mu_{r}\left(\sigma^{\prime} \mid \eta^{\prime}\right)}\right\},
$$

where with a slight abuse of notation we denote the probability density of measures with the same letter. Note that in the sequel $\eta=\mathbf{T} \sigma, \eta^{\prime}=\mathbf{T} \sigma^{\prime}$, if not otherwise stated. A trial state $\sigma^{\prime}$ is generated by the first level and the simple reconstruction with the transition probability

$$
Q\left(\sigma, \sigma^{\prime}\right)=\alpha_{C G}\left(\eta, \eta^{\prime}\right) \bar{\rho}\left(\eta, \eta^{\prime}\right) \mu_{r}\left(\sigma^{\prime} \mid \eta^{\prime}\right), \text { for } \sigma \neq \sigma^{\prime} .
$$

It is easy to check that $Q\left(\sigma, \sigma^{\prime}\right)$ satisfies the detailed balance condition, see Definition 2, with $\mu_{0}(d \sigma)=\bar{\mu}^{(0)}(d \eta) \mu_{r}(d \sigma \mid \eta)$. i.e. $Q\left(\sigma, \sigma^{\prime}\right) \mu_{0}(\sigma)=$ $Q\left(\sigma^{\prime}, \sigma\right) \mu_{0}\left(\sigma^{\prime}\right)$. This property lead to the simplified formulation of the second level acceptance probability (17) from the one suggested by the Metropolis method $\alpha_{f}\left(\sigma, \sigma^{\prime}\right)=\min \left\{1, \frac{\mu\left(\sigma^{\prime}\right) Q\left(\sigma^{\prime}, \sigma\right)}{\mu(\sigma) Q\left(\sigma, \sigma^{\prime}\right)}\right\}$. The probability of moving from a state $\sigma$ to a next $\sigma^{\prime}$ is $\alpha_{f}\left(\sigma, \sigma^{\prime}\right) \alpha_{C G}\left(\eta, \eta^{\prime}\right) \mu_{r}\left(\sigma^{\prime} \mid \eta^{\prime}\right) \bar{\rho}\left(\eta, \eta^{\prime}\right)$. Therefore the method generates a Markov chain $\left\{Y_{n}\right\}$, starting from an arbitrary initial state $\sigma_{0}$, with transition kernel

$$
\begin{gathered}
\mathcal{K}_{C G}\left(\sigma, \sigma^{\prime}\right)=\alpha_{f}\left(\sigma, \sigma^{\prime}\right) \alpha_{C G}\left(\eta, \eta^{\prime}\right) \mu_{r}\left(\sigma^{\prime} \mid \eta^{\prime}\right) \bar{\rho}\left(\eta, \eta^{\prime}\right) \quad \text { for } \sigma \neq \sigma^{\prime} \\
\mathcal{K}_{C G}(\sigma, \sigma)=1-\int_{\Sigma} \alpha_{f}\left(\sigma, \sigma^{\prime}\right) \alpha_{C G}\left(\eta, \eta^{\prime}\right) \mu_{r}\left(\sigma^{\prime} \mid \eta^{\prime}\right) \bar{\rho}\left(\eta, \eta^{\prime}\right) d \sigma^{\prime}
\end{gathered}
$$

We denote $E=\{\sigma \in \Sigma: \mu(\sigma)>0\}$ and $\tilde{E}=\left\{\sigma \in \Sigma: \mu_{0}(\sigma)>0\right\}$ the support of the microscopic and the proposal distributions respectively.

With the following Theorem, the proof of which is given in Appendix A, we prove that transition kernel $\mathcal{K}_{C G}\left(\sigma, \sigma^{\prime}\right)$ satisfies the detailed balance condition, that ensures the method generates samples from the target measure. Furthermore, irreducibility and aperiodicity properties are satisfied that guarantee ergodicity of $\left\{Y_{n}\right\}$, i.e. $\frac{1}{n} \sum_{j=1}^{n} f\left(Y_{j}\right)$ is a convergent approximation of the averages $\int f(\sigma) \mu(d \sigma)$ for any $f \in L^{1}(\mu)$.

Theorem 1. For every conditional distribution $\bar{\rho}\left(\eta, \eta^{\prime}\right)$ on $\bar{\Sigma}$, and $\mu_{r}(\cdot \mid \eta)$ on $\{\sigma \in \Sigma: \mathbf{T} \sigma=\eta\}$

i) The transition kernel $\mathcal{K}_{C G}\left(\sigma, \sigma^{\prime}\right)$ satisfies the detailed balance $(D B)$ condition with $\mu(\sigma)$.

ii) $\mu(\sigma)$ is a stationary distribution of the chain.

iii) If $\bar{\rho}\left(\eta, \eta^{\prime}\right)>0, \mu_{r}(\sigma \mid \eta)>0$ for all $\sigma, \sigma^{\prime} \in E$ and $E \subset \tilde{E}$ holds, then $\left\{Y_{n}\right\}$ is $\mu$-irreducible.

iv) $\left\{Y_{n}\right\}$ is aperiodic. 


\section{Computational Complexity of Multilevel CGMC}

The effectiveness of the multilevel CGMC method is a result of the synthesis of the following two arguments. Firstly, the computational cost of a conventional MH method is reduced by a two-level CGMC method. However this is not enough to prove that the method can indeed accelerate conventional methods, and a mathematical spectral analysis emerges as a key algorithmic need. Therefore, secondly, Theorem 2 provides the relation of the two methods equilibration times using spectral arguments.

\subsection{Computational complexity}

An abstract comparison of the computational complexity of a conventional MH and the two-level CGMC is summarized in Table 1, for sampling a Gibbs measure with Hamiltonian (11). By computational complexity here we mean the cost of calculating energy differences involved at the acceptance probabilities. The following analysis is based on the approximate CG strategy with potential splitting as described in subsection 3.1. Let us consider the canonical ensemble where energy difference is

$$
\Delta H_{N}\left(\sigma, \sigma^{x}\right)=(2 \sigma(x)-1) \sum_{y \in \Lambda_{N}, y \neq x}[K(x-y)+J(x-y) \sigma(y)] .
$$

For potential $J(x-y)$ with interaction range $L$ each particle interacts with a number of $(2 L+1)^{d}$ neighbours and similarly for $K(x-y)$ with range $S$. Therefore the number of operations necessary are $(2 L+1)^{d}+(2 S+1)^{d}$. Similarly the energy differences appearing in the two-level method are

$$
\begin{aligned}
\Delta \bar{H}_{l}^{(0)}\left(\eta, \eta^{k}\right) & =\sum_{l \in \bar{\Lambda}_{M}, k \neq l} \bar{J}(k, l) \eta(l)+\bar{J}(0,0)(\eta(k)-1), \\
\Delta H_{s}\left(\sigma, \sigma^{x}\right) & =(2 \sigma(x)-1) \sum_{y \in \Lambda_{N}, y \neq x} K(x-y) \sigma(y),
\end{aligned}
$$

at the first and second level respectively. The compressed interaction potential $\bar{J}(k, l)$ has the reduced range $L / q$ and the number of operations for calculating $\Delta \bar{H}_{l}^{(0)}\left(\eta, \eta^{k}\right)$ is $(2 L+1)^{d} / Q$, while for $\Delta H_{s}\left(\sigma, \sigma^{x}\right)$ is $(2 S+1)^{d} / Q$. The method, in addition to the reduction of operations due to range suppression, exhibits a computational reduction as a result of the fact that rejected trials at the first level will not be tested at the second, and the calculation of differences $\Delta H_{s}\left(\sigma, \sigma^{x}\right)$ is avoided. A summary of this discussion appears in 
Table 1: Operations count for evaluating energy differences for $n$ iterations. The total number of accepted coarse trials $m$ is the number of the second level iterations tested.

\begin{tabular}{lc}
\hline Method & Operations \\
\hline Metropolis Hastings & $n \times(2 L+1)^{d}+n \times(2 S+1)^{d}$ \\
Two-level CGMC & $n \times(2 L+1)^{d} / Q+m \times(2 S+1)^{d}$ \\
\hline
\end{tabular}

Table 1. When the correction terms $\Delta H_{l}\left(\sigma, \sigma^{x}\right)-\Delta \bar{H}_{l}^{(0)}\left(\eta, \eta^{k}\right)$ are present the additional computational cost in the second level is small, dependent on the decay of rate of $J(x-y)-\bar{J}(k, l), x, y \in \Sigma_{N}, x \in C_{k}, y \in C_{l}$. Since this term is in principle fast decaying, in implementations we can neglect interactions with distance larger than a cut-off range $L_{c}<L$ with a small error, that will contribute further in the computational time reduction.

\subsection{Comparison of equilibration times}

A direct estimation of rate of convergence of a Markov chain generated by a Monte Carlo method is model dependent and in general intractable. Thus for the purpose of this work it is natural to study this property as a comparison of the proposed method with a conventional method. Theorem 2 provides such a comparison of the spectral gap between the conventional $\mathrm{MH}$ method and the two-level CGMC method, for sampling a measure $\mu(d \sigma)$ on a countable state space $\Sigma$. This comparison is summarised in inequality (20) proving that their relation, in terms the spectral gap, is controlled by the approximation $\bar{\mu}^{(0)}(d \eta)$ of marginals $\bar{\mu}(d \eta)=\mu \circ \mathbf{T}^{-1}(d \eta)$.

For a discrete time Markov chain $\left\{X_{n}\right\}$ with transition kernel $\mathcal{K}$ and stationary distribution $\mu$, the mixing time $\tau$ is defined as

$$
\tau:=\min _{n}\left\{\forall \sigma \in \Sigma:\left\|\mathcal{K}^{n}(\sigma, \cdot)-\mu(\cdot)\right\|_{T V} \leq \frac{1}{4}\right\} .
$$

For two probability measures $\mu, \nu$ the total variation norm is $\|\mu-\nu\|_{T V}=$ $\frac{1}{2} \sum_{\sigma}|\mu(\sigma)-\nu(\sigma)|$. Bounds of the total variation norm appearing can be given in terms of $\mathcal{K}$ 's spectral gap $\lambda$, for example for a reversible kernel holds [8]

$$
2\left\|\mathcal{K}^{n}(\sigma, \cdot)-\mu\right\|_{T V} \leq \frac{1}{\mu(\sigma)^{1 / 2}}(1-\lambda)^{n} .
$$

The spectral gap of a kernel $\mathcal{K}$ is defined by $\lambda(\mathcal{K})=\min \left\{\frac{\mathcal{E}(f, f)}{\operatorname{Var}(f)} ; \operatorname{Var}(f) \neq 0\right\}$ with the Dirichlet form $\left.\mathcal{E}(f, f)=\frac{1}{2} \sum_{\sigma, \sigma^{\prime}} \mid f(\sigma)-f \sigma^{\prime}\right)\left.\right|^{2} \mathcal{K}\left(\sigma, \sigma^{\prime}\right) \mu(\sigma)$ and the 
variance $\operatorname{Var}(f)=\frac{1}{2} \sum_{\sigma, \sigma^{\prime}}\left|f(\sigma)-f\left(\sigma^{\prime}\right)\right|^{2} \mu\left(\sigma^{\prime}\right) \mu(\sigma)$. In view of (19), one can say that between two algorithms producing Markov chains with identical equilibrium distributions better in terms of speed of convergence is the one with the larger spectral gap.

Let $\mathcal{E}\left(\mathcal{K}_{C G}\right), \mathcal{E}\left(\mathcal{K}_{c}\right)$ denote the Dirichlet forms and $\lambda\left(\mathcal{K}_{C G}\right), \lambda\left(\mathcal{K}_{c}\right)$ the spectral gap corresponding to the two-level CGMC and the classical Metropolis transition kernels $\mathcal{K}_{C G}\left(\sigma, \sigma^{\prime}\right)$ (18) and $\mathcal{K}_{c}\left(\sigma, \sigma^{\prime}\right)$ (12) respectively.

Theorem 2. Let $\rho\left(\sigma, \sigma^{\prime}\right)$ be a symmetric proposal transition probability for the conventional $M H$ algorithm and $\bar{\rho}\left(\eta, \eta^{\prime}\right)$ a symmetric proposal transition probability on the coarse space $\bar{\Sigma}$ for the two-level CGMC algorithm, then for any conditional probability $\mu_{r}(\sigma \mid \eta)$

$$
\mathcal{A} \underline{\gamma} \lambda\left(\mathcal{K}_{c}\right) \leq \lambda\left(\mathcal{K}_{C G}\right) \leq \bar{\gamma} \lambda\left(\mathcal{K}_{c}\right)
$$

where $\mathcal{A}=\inf _{\sigma, \sigma^{\prime}}\left\{\mathcal{A}\left(\sigma, \sigma^{\prime}\right)\right\}$ and $\underline{\gamma}>0, \bar{\gamma}>0$ such that $\underline{\gamma} \leq \mathcal{B}\left(\sigma, \sigma^{\prime}\right) \leq \bar{\gamma}$, with $\mathcal{A}\left(\sigma, \sigma^{\prime}\right)$ and $\mathcal{B}\left(\sigma, \sigma^{\prime}\right)$ defined in lemma (3).

\section{Remarks.}

1. Existence of finite and positive values of $\mathcal{A}=\inf _{\sigma, \sigma^{\prime}}\left\{\mathcal{A}\left(\sigma, \sigma^{\prime}\right)\right\}$ is ensured for the models studied in this work. Consider the Gibbs measure $\mu_{N, \beta}(d \sigma) \propto \exp \left\{-\beta H_{N}(\sigma)\right\}$ and $\bar{\mu}_{M, \beta}^{(0)}(d \eta) \propto \exp \left\{-\beta \bar{H}^{(0)}(\eta)\right\}$ as defined in Section 2, Let $J(r)$, s.t. $\sum_{r} J(r)=J^{*}<\infty$ with the com-

pressed interactiosn $\bar{J}$ as defined in ([6) $)$. All possible values of $\mathcal{A}\left(\sigma, \sigma^{\prime}\right)$, (23) , are $\mathcal{A}\left(\sigma, \sigma^{\prime}\right)=1$,

$$
\mathcal{A}\left(\sigma, \sigma^{\prime}\right)=\min \left\{e^{-\beta \Delta \bar{H}^{(0)}\left(\eta, \eta^{\prime}\right)}, e^{-\beta \Delta \bar{H}^{(0)}\left(\eta^{\prime}, \eta\right)}\right\} \geq e^{-\beta \bar{J}^{*}} .
$$

and

$$
\begin{aligned}
\mathcal{A}\left(\sigma, \sigma^{\prime}\right) & =\min \left\{e^{-\beta\left(\Delta H_{N}\left(\sigma, \sigma^{\prime}\right)-\Delta \bar{H}^{(0)}\left(\eta, \eta^{\prime}\right)\right)}, e^{-\beta\left(\Delta H_{N}\left(\sigma^{\prime}, \sigma\right)-\Delta \bar{H}^{(0)}\left(\eta^{\prime}, \eta\right)\right)}\right\} \\
& =1+\mathcal{O}\left(\frac{Q}{(2 L+1)^{d}}\right)
\end{aligned}
$$

This is a result of the known estimate Lemma (2.3) [20],

$$
\Delta H_{N}\left(\sigma, \sigma^{\prime}\right)-\Delta \bar{H}^{(0)}(\eta)=\mathcal{O}\left(\frac{Q}{(2 L+1)^{d}}\right)
$$

This proves also that values of $\mathcal{A}\left(\sigma, \sigma^{\prime}\right)$ are close to 1 controlled by the approximation parameter $Q$. 
2. Term $\mathcal{B}\left(\sigma, \sigma^{\prime}\right)$ depends only on the difference between the proposal kernels in the two methods. For example if $\mu_{r}(\sigma \mid \eta)$ is chosen such that $\rho\left(\sigma, \sigma^{\prime}\right)=\bar{\rho}\left(\eta, \eta^{\prime}\right) \mu_{r}\left(\sigma^{\prime} \mid \eta^{\prime}\right)$ for all $\sigma, \sigma^{\prime} \in \Sigma$ then $\mathcal{B}\left(\sigma, \sigma^{\prime}\right) \equiv 1$, and inequality (20) depends only on $\mathcal{A}$, that is

$$
\mathcal{A} \lambda\left(\mathcal{K}_{c}\right) \leq \lambda\left(\mathcal{K}_{C G}\right) \leq \lambda\left(\mathcal{K}_{c}\right)
$$

Numerically the statement of the Theorem is revealed by a comparison of the average acceptance probabilities in examples, see Figures 4 . We expect that best approach in terms of equilibration rate is a rejection free method for sampling the CG distribution, which is an approach that we elaborate with in a forthcoming work.

To prove Theorem 2 we need the following lemmata.

Lemma 3. For symmetric proposal transition kernels $\rho\left(\sigma, \sigma^{\prime}\right)=\rho\left(\sigma^{\prime}, \sigma\right)$ and $\bar{\rho}\left(\eta, \eta^{\prime}\right)=\bar{\rho}\left(\eta^{\prime}, \eta\right)$, for all $\sigma, \sigma^{\prime} \in \Sigma$ with $\sigma \neq \sigma^{\prime}$,

$$
\mathcal{K}_{C G}\left(\sigma, \sigma^{\prime}\right)=\mathcal{A}\left(\sigma, \sigma^{\prime}\right) \mathcal{B}\left(\sigma, \sigma^{\prime}\right) \mathcal{K}_{c}\left(\sigma, \sigma^{\prime}\right)
$$

where

$$
\mathcal{B}\left(\sigma, \sigma^{\prime}\right)=\frac{\bar{\rho}\left(\eta, \eta^{\prime}\right)}{\rho\left(\sigma, \sigma^{\prime}\right)}\left\{\begin{array}{ll}
\mu_{r}\left(\sigma^{\prime} \mid \eta^{\prime}\right), & \text { if } \alpha_{f}\left(\sigma, \sigma^{\prime}\right)=1 \\
\mu_{r}(\sigma \mid \eta), & \text { if } \alpha_{f}\left(\sigma, \sigma^{\prime}\right)<1
\end{array} .\right.
$$

Furthermore we define the subsets

$$
\begin{aligned}
& C_{1}=\left\{\left(\sigma, \sigma^{\prime}\right) \in \Sigma \times \Sigma:\left\{\alpha<1, \alpha_{C G}<1, \alpha_{f}<1\right\} \quad \text { or }\left\{\alpha=1, \alpha_{C G}=1, \alpha_{f}=1\right\}(22)\right. \\
& C_{2}=\left\{\left(\sigma, \sigma^{\prime}\right) \in \Sigma \times \Sigma:\left\{\alpha=1, \alpha_{C G}<1, \alpha_{f}=1\right\} \text { or }\left\{\alpha<1, \alpha_{C G}=1, \alpha_{f}<1\right\}\right\} \\
& C_{3}=\left\{\left(\sigma, \sigma^{\prime}\right) \in \Sigma \times \Sigma:\left\{\alpha=1, \alpha_{C G}=1, \alpha_{f}<1\right\} \text { or }\left\{\alpha<1, \alpha_{C G}<1, \alpha_{f}=1\right\}\right\} \\
& C_{4}=\left\{\left(\sigma, \sigma^{\prime}\right) \in \Sigma \times \Sigma:\left\{\alpha<1, \alpha_{C G}=1, \alpha_{f}=1\right\} \text { or }\left\{\alpha=1, \alpha_{C G}<1, \alpha_{f}<1\right\}\right\}
\end{aligned}
$$

and

$$
\mathcal{A}\left(\sigma, \sigma^{\prime}\right)= \begin{cases}1, & \text { if }\left(\sigma, \sigma^{\prime}\right) \in C_{1} \\ \min \left\{\frac{\bar{\mu}^{(0)}\left(\eta^{\prime}\right)}{\bar{\mu}^{(0)}(\eta)}, \frac{\bar{\mu}^{(0)}(\eta)}{\bar{\mu}^{(0)}\left(\eta^{\prime}\right)}\right\}, & \text { if }\left(\sigma, \sigma^{\prime}\right) \in C_{2} \\ \min \left\{\frac{\mu\left(\sigma^{\prime}\right) \bar{\mu}^{(0)}(\eta)}{\mu(\sigma) \bar{\mu}^{(0)}\left(\eta^{\prime}\right)}, \frac{\mu(\sigma) \bar{\mu}^{(0)}\left(\eta^{\prime}\right)}{\mu\left(\sigma^{\prime}\right) \bar{\mu}^{(0)}(\eta)}\right\}, & \text { if }\left(\sigma, \sigma^{\prime}\right) \in C_{3} \\ \min \left\{\frac{\mu\left(\sigma^{\prime}\right)}{\mu(\sigma)}, \frac{\mu(\sigma)}{\mu\left(\sigma^{\prime}\right)}\right\}, & \text { if }\left(\sigma, \sigma^{\prime}\right) \in C_{4}\end{cases}
$$

Proof. Recall that $\mathcal{K}_{c}\left(\sigma, \sigma^{\prime}\right)=\alpha\left(\sigma, \sigma^{\prime}\right) \rho\left(\sigma, \sigma^{\prime}\right)$, for $\sigma \neq \sigma^{\prime}$, which for the conventional $\mathrm{MH}$ method and the hypothesis of symmetry of $\rho$ becomes 
$\mathcal{K}_{c}\left(\sigma, \sigma^{\prime}\right)=\min \left\{1, \frac{\mu\left(\sigma^{\prime}\right)}{\mu(\sigma)}\right\} \rho\left(\sigma, \sigma^{\prime}\right)$. Similarly the two-level CGMC kernel becomes

$$
\begin{aligned}
\mathcal{K}_{C G}\left(\sigma, \sigma^{\prime}\right)= & \min \left\{1, \frac{\mu\left(\sigma^{\prime}\right) \bar{\mu}^{(0)}(\eta) \mu_{r}(\sigma \mid \eta)}{\mu(\sigma) \bar{\mu}^{(0)}\left(\eta^{\prime}\right) \mu_{r}\left(\sigma^{\prime} \mid \eta^{\prime}\right)}\right\} \\
& \times \min \left\{1, \frac{\bar{\mu}^{(0)}\left(\eta^{\prime}\right)}{\bar{\mu}^{(0)}(\eta)}\right\} \bar{\rho}\left(\eta, \eta^{\prime}\right) \mu_{r}\left(\sigma^{\prime} \mid \eta^{\prime}\right) .
\end{aligned}
$$

The proof is based on a case study on the values of the acceptance probabilities $\alpha\left(\sigma, \sigma^{\prime}\right)=\min \left\{1, \frac{\mu\left(\sigma^{\prime}\right)}{\mu(\sigma)}\right\}, \alpha_{f}\left(\sigma, \sigma^{\prime}\right)=\min \left\{1, \frac{\mu\left(\sigma^{\prime}\right) \bar{\mu}^{(0)}(\eta) \mu_{r}(\sigma \mid \eta)}{\mu(\sigma) \bar{\mu}^{(0)}\left(\eta^{\prime}\right) \mu_{r}\left(\sigma^{\prime} \mid \eta^{\prime}\right)}\right\}$ and $\alpha_{C G}\left(\eta, \eta^{\prime}\right)=\min \left\{1, \frac{\bar{\mu}^{(0)}\left(\eta^{\prime}\right)}{\bar{\mu}^{(0)}(\eta)}\right\}$. In the following we also use extensively the symmetry property of $\rho$ and $\bar{\rho}$. All possible combinations on their values are categorized in the sets $C_{i}, i=1, \ldots, 4$ defined in (22). For $\left(\sigma, \sigma^{\prime}\right) \in C_{1}$ and $\alpha\left(\sigma, \sigma^{\prime}\right)<1, \alpha_{f}\left(\sigma, \sigma^{\prime}\right)<1$ and $\alpha_{C G}\left(\eta, \eta^{\prime}\right)<1, \mathcal{K}_{c}\left(\sigma, \sigma^{\prime}\right)=\frac{\mu\left(\sigma^{\prime}\right)}{\mu(\sigma)} \rho\left(\sigma, \sigma^{\prime}\right)$ and

$$
\mathcal{K}_{C G}\left(\sigma, \sigma^{\prime}\right)=\frac{\mu\left(\sigma^{\prime}\right) \bar{\mu}^{(0)}(\eta)}{\mu(\sigma) \bar{\mu}^{(0)}\left(\eta^{\prime}\right)} \frac{\bar{\mu}^{(0)}\left(\eta^{\prime}\right)}{\bar{\mu}^{(0)}(\eta)} \bar{\rho}\left(\eta, \eta^{\prime}\right) \mu_{r}\left(\sigma^{\prime} \mid \eta^{\prime}\right)=\frac{\mu\left(\sigma^{\prime}\right)}{\mu(\sigma)} \bar{\rho}\left(\eta, \eta^{\prime}\right) \mu_{r}\left(\sigma^{\prime} \mid \eta^{\prime}\right)
$$

and we derive their relation $\mathcal{K}_{C G}\left(\sigma, \sigma^{\prime}\right)=\frac{\bar{\rho}\left(\eta, \eta^{\prime}\right) \mu_{r}(\sigma \mid \eta)}{\rho\left(\sigma, \sigma^{\prime}\right)} \mathcal{K}_{c}\left(\sigma, \sigma^{\prime}\right)$. With similar simple calculations, that we omit here, for all sub-cases that are encountered in subsets $C_{i}, i=1, \ldots 4$ we have, for $\left(\sigma, \sigma^{\prime}\right) \in C_{1}$

$$
\mathcal{K}_{C G}\left(\sigma, \sigma^{\prime}\right)=\mathcal{B}\left(\sigma, \sigma^{\prime}\right) \mathcal{K}_{c}\left(\sigma, \sigma^{\prime}\right)
$$

with

$$
\mathcal{B}\left(\sigma, \sigma^{\prime}\right)=\frac{\bar{\rho}\left(\eta, \eta^{\prime}\right)}{\rho\left(\sigma, \sigma^{\prime}\right)} \begin{cases}\mu_{r}\left(\sigma^{\prime} \mid \eta^{\prime}\right), & \text { if } \alpha_{f}\left(\sigma, \sigma^{\prime}\right)=1 \\ \mu_{r}(\sigma \mid \eta), & \text { if } \alpha_{f}\left(\sigma, \sigma^{\prime}\right)<1\end{cases}
$$

For $\left(\sigma, \sigma^{\prime}\right) \in C_{2}$ such that $\alpha\left(\sigma, \sigma^{\prime}\right)=1, \alpha_{C G}\left(\eta, \eta^{\prime}\right)<1, \alpha_{f}\left(\sigma, \sigma^{\prime}\right)=1$,

$$
\mathcal{K}_{C G}\left(\sigma, \sigma^{\prime}\right)=\frac{\bar{\mu}^{(0)}\left(\eta^{\prime}\right)}{\bar{\mu}^{(0)}(\eta)} \frac{\bar{\rho}\left(\eta, \eta^{\prime}\right) \mu_{r}\left(\sigma^{\prime} \mid \eta^{\prime}\right)}{\rho\left(\sigma, \sigma^{\prime}\right)} \mathcal{K}_{c}\left(\sigma, \sigma^{\prime}\right)
$$

and for $\alpha\left(\sigma, \sigma^{\prime}\right)<1, \alpha_{C G}\left(\eta, \eta^{\prime}\right)=1, \alpha_{f}\left(\sigma, \sigma^{\prime}\right)<1$,

$$
\mathcal{K}_{C G}\left(\sigma, \sigma^{\prime}\right)=\frac{\bar{\mu}^{(0)}(\eta)}{\bar{\mu}^{(0)}\left(\eta^{\prime}\right)} \frac{\bar{\rho}\left(\eta, \eta^{\prime}\right) \mu_{r}(\sigma \mid \eta)}{\rho\left(\sigma, \sigma^{\prime}\right)} K_{c}\left(\sigma, \sigma^{\prime}\right)
$$


that we can summarize to

$$
\mathcal{K}_{C G}\left(\sigma, \sigma^{\prime}\right)=\min \left\{\frac{\bar{\mu}^{(0)}(\eta)}{\bar{\mu}^{(0)}\left(\eta^{\prime}\right)}, \frac{\bar{\mu}^{(0)}\left(\eta^{\prime}\right)}{\bar{\mu}^{(0)}(\eta)}\right\} \mathcal{B}\left(\sigma, \sigma^{\prime}\right) K_{c}\left(\sigma, \sigma^{\prime}\right),
$$

since for example in the first case $\frac{\bar{\mu}^{(0)}\left(\eta^{\prime}\right)}{\bar{\mu}^{(0)}(\eta)}<1<\frac{\bar{\mu}^{(0)}(\eta)}{\bar{\mu}^{(0)}\left(\eta^{\prime}\right)}$ and inversely for the second. Following the same reasoning for $\left(\sigma, \sigma^{\prime}\right) \in C_{3}$,

$$
\mathcal{K}_{C G}\left(\sigma, \sigma^{\prime}\right)=\min \left\{\frac{\mu\left(\sigma^{\prime}\right) \bar{\mu}^{(0)}(\eta)}{\mu(\sigma) \bar{\mu}^{(0)}\left(\eta^{\prime}\right)}, \frac{\mu(\sigma) \bar{\mu}^{(0)}\left(\eta^{\prime}\right)}{\mu\left(\sigma^{\prime}\right) \bar{\mu}^{(0)}(\eta)}\right\} \mathcal{B}\left(\sigma, \sigma^{\prime}\right) \mathcal{K}_{c}\left(\sigma, \sigma^{\prime}\right),
$$

and for $\left(\sigma, \sigma^{\prime}\right) \in C_{4}$

$$
\mathcal{K}_{C G}\left(\sigma, \sigma^{\prime}\right)=\min \left\{\frac{\mu\left(\sigma^{\prime}\right)}{\mu(\sigma)}, \frac{\mu(\sigma)}{\mu\left(\sigma^{\prime}\right)}\right\} \mathcal{B}\left(\sigma, \sigma^{\prime}\right) \mathcal{K}_{c}\left(\sigma, \sigma^{\prime}\right) .
$$

All these steps prove, the following relation of transition kernels generated by Algorithms 1 and 2 ,

$$
\mathcal{K}_{C G}\left(\sigma, \sigma^{\prime}\right)=\mathcal{A}\left(\sigma, \sigma^{\prime}\right) \mathcal{B}\left(\sigma, \sigma^{\prime}\right) \mathcal{K}_{c}\left(\sigma, \sigma^{\prime}\right),
$$

with $\mathcal{A}\left(\sigma, \sigma^{\prime}\right)$ and $\mathcal{B}\left(\sigma, \sigma^{\prime}\right)$ defined in (23) and (21).

The proof of Theorem 2 is based on the application of Lemma 3 and Lemma 3.3 in the work of P. Diaconis and L. Saloff-Coste [8] that is stated here for completeness.

Lemma 4. Let $\mathcal{K}, \mu$ and $\mathcal{K}^{\prime}, \mu^{\prime}$ be two Markov chains on the same finite set $X$. Assume that there exist $A, a>0$ such that

$$
\mathcal{E}^{\prime} \leq A \mathcal{E}, \quad a \mu \leq \mu^{\prime}
$$

then

$$
\lambda^{\prime} \leq \frac{A}{a} \lambda .
$$

We conclude with the proof of Theorem 2,

Proof. We compare the Dirichlet forms $\mathcal{E}\left(\mathcal{K}_{C G}\right), \mathcal{E}\left(\mathcal{K}_{c}\right)$ using the definition of Dirichlet form and applying Lemma (3) . By the definition of $\mathcal{A}\left(\sigma, \sigma^{\prime}\right)$ for all $\sigma, \sigma^{\prime} \in \Sigma$ holds $0<\mathcal{A}\left(\sigma, \sigma^{\prime}\right) \leq 1$ and assume that $\mathcal{A}:=\inf _{\sigma, \sigma^{\prime}}\left\{\mathcal{A}\left(\sigma, \sigma^{\prime}\right)\right\}>0$. Then by Lemma 3

$$
\inf _{\sigma, \sigma^{\prime}}\left\{\mathcal{A}\left(\sigma, \sigma^{\prime}\right)\right\} \mathcal{B}\left(\sigma, \sigma^{\prime}\right) \mathcal{K}_{c}\left(\sigma, \sigma^{\prime}\right) \leq \mathcal{K}_{C G}\left(\sigma, \sigma^{\prime}\right) \leq \mathcal{B}\left(\sigma, \sigma^{\prime}\right) \mathcal{K}_{c}\left(\sigma, \sigma^{\prime}\right)
$$


Let $\underline{\gamma}>0$ and $\bar{\gamma}>0$ such that $\underline{\gamma} \leq \mathcal{B}\left(\sigma, \sigma^{\prime}\right) \leq \bar{\gamma}$. Then

$$
\inf _{\sigma, \sigma^{\prime}}\left\{\mathcal{A}\left(\sigma, \sigma^{\prime}\right)\right\} \underline{\gamma}_{c}\left(\sigma, \sigma^{\prime}\right) \leq \mathcal{K}_{C G}\left(\sigma, \sigma^{\prime}\right) \leq \bar{\gamma} \mathcal{K}_{c}\left(\sigma, \sigma^{\prime}\right)
$$

Recalling the definition of Dirichlet form for kernel $\mathcal{K}_{C G}$,

$$
\mathcal{E}_{C G}(f, f)=\frac{1}{2} \sum_{\sigma, \sigma^{\prime}}\left|f(\sigma)-f\left(\sigma^{\prime}\right)\right|^{2} \mathcal{K}_{C G}\left(\sigma, \sigma^{\prime}\right) \mu(\sigma),
$$

and the above relation we can write $\inf \left\{\mathcal{A}\left(\sigma, \sigma^{\prime}\right)\right\} \underline{\gamma} \mathcal{E}_{c} \leq \mathcal{E}_{C G} \leq \bar{\gamma} \mathcal{E}_{c}$. Application of Lemma 4, for which here $\mu^{\prime} \equiv \mu$, thus $a=1$ and $A=\inf _{\sigma, \sigma^{\prime}}\left\{\mathcal{A}\left(\sigma, \sigma^{\prime}\right)\right\} \underline{\gamma}$ for the left hand side inequality, and $A=\bar{\gamma}$ for the right hand side, gives the relation of spectral gaps

$$
\inf _{\sigma, \sigma^{\prime}}\left\{\mathcal{A}\left(\sigma, \sigma^{\prime}\right)\right\} \underline{\gamma} \lambda\left(\mathcal{K}_{c}\right) \leq \lambda\left(\mathcal{K}_{C G}\right) \leq \bar{\gamma} \lambda\left(\mathcal{K}_{c}\right)
$$

\section{Benchmark examples for the canonical ensemble}

\subsection{Combined Ising and Curie Weiss model}

We consider a benchmark example of competing short- and long- range interactions, where constants $\mathcal{A}$, and $\gamma, \bar{\gamma}$ appearing in Theorem 2 are calculated explicitly. Furthermore analytical expressions for the free energy in the thermodynamic limit, $N \rightarrow \infty$, are known [16]. The energy of the system at configuration $\sigma \in \Sigma_{N}$ is

$$
\begin{aligned}
H_{N}(\sigma) & =-\frac{K}{2} \sum_{x \in \Lambda_{N}} \sum_{|x-y|=1} \sigma(x) \sigma(y)-\frac{J}{2 N} \sum_{x \in \Lambda_{N}} \sum_{y \neq x} \sigma(x) \sigma(y)-h \sum_{x \in \Lambda_{N}} \sigma(x) \\
& =H_{s}(\sigma)+H_{l}(\sigma)-h \sum_{x \in \Lambda_{N}} \sigma(x) .
\end{aligned}
$$

The interactions involved in $H_{s}(\sigma)$ are of the nearest-neighbour type with strength $K$, while $H_{l}(\sigma)$ represents a mean-field approximation of a potential $J(x-y)$ as in (8) for example, averaged over all lattice sites. The coarse grained Hamiltonian $\bar{H}_{l}(\eta)$ is exact, equal to the microscopic energy $H_{l}(\sigma)$. Indeed, for any coarse graining level $Q$

$$
H_{l}(\sigma)=\bar{H}_{l}(\eta)=-\frac{J}{2 N} \sum_{k \in \bar{\Lambda}_{M}} \sum_{l \in \bar{\Lambda}_{M} l \neq k} \eta(k) \eta(l)-\frac{J}{2 N} \sum_{k \in \bar{\Lambda}_{M}} \eta(k)(\eta(k)-1)
$$


where $\eta(k)=\sum_{x \in C_{k}} \sigma(x), k \in \bar{\Lambda}_{M}$. No coarse graining approximation error is involved from compressing long range interactions, that allows us to study the effect of the splitting of short- and long- range interactions. We consider the canonical ensemble with the spin-flip dynamics.

The detailed calculations on the application of Theorem 2 are given in Appendix B, where we prove that the constants appearing in inequality (20) are

$$
\begin{gathered}
\underline{\gamma}=\bar{\gamma}=1, \text { and } \\
\mathcal{A}= \begin{cases}\min \left\{e^{-|J|}, e^{-2|K|}\right\} & \text { for } K \neq 0, \\
1 & \text { for } K=0 .\end{cases}
\end{gathered}
$$

The relation of the spectral gap of the methods is, for $K \neq 0$,

$$
\min \left\{e^{-|J|}, e^{-2|K|}\right\} \lambda\left(\mathcal{K}_{c}\right) \leq \lambda\left(\mathcal{K}_{C G}\right) \leq \lambda\left(\mathcal{K}_{c}\right) .
$$

For $K=0$ Theorem 2 verifies the equivalence of the two methods $\lambda\left(\mathcal{K}_{C G}\right)=$ $\lambda\left(\mathcal{K}_{c}\right)$ as is expected. The dependence on the splitting of energy is revealed, showing that the governing parameter is the relative strength of the short and long range interactions $J$ and $K$.

Here we present simulation results for two dimensional lattice systems, while in [15] one can find a detailed numerical study for one-dimensional systems. Table 2 shows a comparison of the computational time between the $\mathrm{MH}$ and the two-level CGMC method for a lattice $N=16 \times 16$ and coarsening parameter $Q=q \times q$, for $q=4$ and $q=8$. The results depict a reduce of computational effort at a rate close to $\mathcal{O}(Q)$. This rate is not exactly $\mathcal{O}(Q)$ because of the additional computational time necessary for implementing the local uniform reconstruction. The hysteresis diagram is indicated in Figure 2, for the average total coverage $<c>$ first upon increasing the field $h$ from low values and then decreasing it from high values. The total coverage is $c(\sigma)=N^{-1} \sum_{x} \sigma(x)$ and average quantities $\langle c\rangle$ are computed after equilibration in the Monte Carlo sampling, using 1000 samples. The tests demonstrate that the two-level CGMC method predicts correctly the phase transition regime, compared to the conventional $\mathrm{MH}$, with a reduced computational cost.

\subsection{Kac type interactions}

Known error estimates indicate that the potential decay is one of the parameters controlling the approximation error in coarse graining techniques. 
Table 2: CPU cost comparisons for different resolutions in hysteresis simulations. $K=$ $1, J=5, h \in[0,6], N=16 \times 16$.

\begin{tabular}{lc}
\hline Method & CPU (min) \\
\hline MH q=1 & 327 \\
Two-level CGMC q=4 & 28 \\
Two-level CGMC q=8 & 9 \\
\hline
\end{tabular}

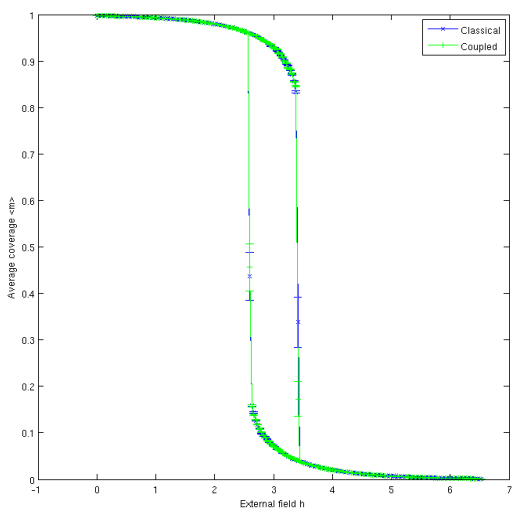

Figure 2: Comparison of hysteresis of the microscopic with the two-level process for model 5.1. $N=64 \times 64, q=4, K=1, J=5$.

The effect of potential singularities on the coarsening error and improving strategies encountering multi-body interactions and/or potential splitting has been studied in [2]. The example tested in this section is chosen to study the potential splitting strategy with the proposed method, that is shown to improve direct CGMC results. We consider a long range Kac type interaction potential $J(r)=N^{-1} V(r / N), r=|x-y|>0, x, y \in \Lambda_{N}$ with an algebraic decay

$$
V(r)=\frac{v}{r^{3 / 2}}, \text { if } r>0,
$$

where constant $v$ is chosen to ensure the conservation of the total mass $J_{0}=$ $\int J(r) d r$. The potential splitting strategy is applied by decomposing the interacting potential into a short-range $J_{s}(r)$, as well as a long-range $J_{l}(r)$, 
defined by

$$
J_{s}(r)= \begin{cases}J(r) & \text { for } 0<r \leq S \\ 0 & \text { for } S<r\end{cases}
$$

and

$$
J_{l}(r)=J(r)-J_{s}(r) .
$$

This splitting defines the energy of teh system in the form $H_{N}(\sigma)=H_{s}(\sigma)+$ $H_{l}(\sigma)$ as in (11) with $K(x-y)=J_{s}(|x-y|)$ and $J(x-y)=J_{l}(|x-y|)$. In Figure 3 and Table 3 we present simulation results in the canonical ensemble derived from the three methods, the conventional $\mathrm{MH}$ with interaction potential $J(|x-y|), x, y \in \Lambda_{N}$ and, the two-level CGMC with interactions $\bar{J}_{l}(k, l), k, l \in \bar{\Lambda}_{M}$ and $J_{s}(|x-y|)$, sampling on the microscopic space $\Sigma$, and the CGMC with interaction potential $\bar{J}$ sampling on the coarse space $\bar{\Sigma}_{M}$. It is observed that, for a fixed coarsening parameter $Q$, the two-level method reduces the coarse graining error of CGMC simulations, since the most singular part of $J(r), J_{s}(r)$, is treated in the microscopic space and coarsening is applied only to its fast decaying part $J_{l}(r)$, Figure 3. Figure 4 demonstrates the increase of acceptance rate of Metropolis sampling when viewing the proposed method as an improving the propoposed samples. Overall, as is stated in Theorem 2, the method's acceptance rate is comparable to the conventional method. Additionally, a reduction of the computational time compared to the conventional sampling method is achieved. The error ap-

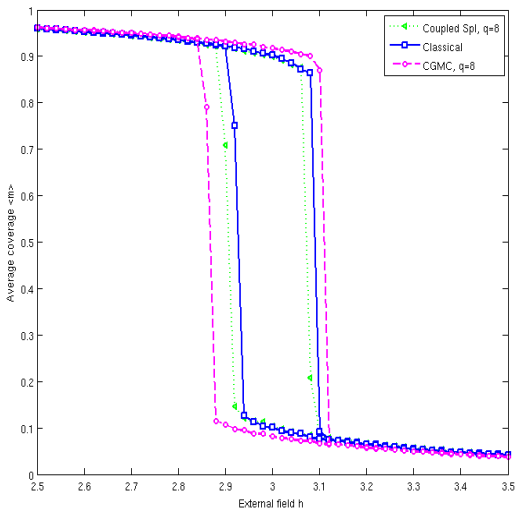

Figure 3: Comparison of hysteresis of the microscopic with the compressed (CGMC) and the two-level (ML CGMC) process for model 5.2. $\beta J_{0}=6, N=512, q=8, S=1$. 


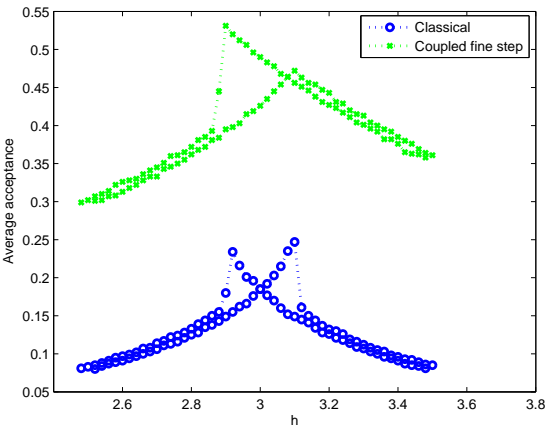

(a)

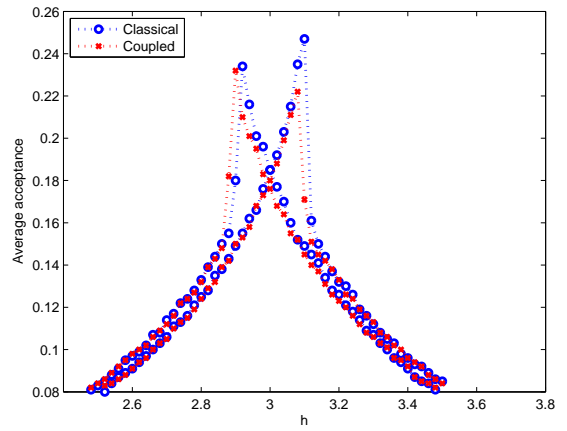

(b)

Figure 4: (a) The second step ML CGMC and the conventional MH average acceptance probabilities in hysteresis simulations, Figure 3 showing increase of acceptances in a Metropolis - Hastings method. (b) Comparison of the total ML CGMC and the conventional $\mathrm{MH}$ average acceptance showing equivalent equilibration rates.

pearing in Table 3 is the $l^{2}$ distance of the average total coverage $\langle c\rangle$, of the indicated method, from the conventional $\mathrm{MH}$ result, as a function of the external field $h$.

Table 3: CPU cost and error comparisons for different resolutions and methods. $N=$ $512, \beta J_{0}=6, S=1$.

\begin{tabular}{lcc}
\hline Method & CPU time & Error \\
\hline MH q=1 & 252 & 0 \\
CGMC q=8 & 47 & 0.78 \\
Two-level CGMC q=8 & 57 & 0.65 \\
CGMC q=64 & 5 & 2.10 \\
Two-level CGMC q=64 & 6 & 0.81 \\
\hline
\end{tabular}

\section{Nanopattern formation in heteroepitaxy - Microcanonical en- semble}

We study a nanopattern formation problem and show how the proposed method can provide microscopic information, benefiting from the low computational cost of the coarse graining technique while refining the error introduced. The system studied here is characterized by the interplay of short 
ranged attraction and long ranged repulsion interactions between particles. Such an interplay can lead to the formation of patterns, such as discs and stripes appearing for example in heteroepitaxy.

The energy of the system is given by $H_{N}(\sigma)$, as in (2), with isotropic interaction potential

$$
J(r)=J_{0}\left(e^{-\left(r / r_{a}\right)^{2}}-\chi e^{-\left(r / r_{r}\right)^{2}}\right), r=|x-y|>0, x, y \in \Lambda_{N} .
$$

$J_{0}$ is the strength of the potential, $r_{a}$ and $r_{r}$ are the dimensionless length scales of attraction and repulsion, respectively, and $\chi$ is the repulsion strength parameter. A study of the kinetic phase diagrams and application of CGMC methods for this system is given in [5]. The underlying dynamics describing the surface diffusion of particles considered are spin-exchange, obeying the exclusion principle and conserving the order parameter microcanonically. The order parameter here is the total coverage $c_{0}=N^{-1} \sum_{x \in \Lambda_{N}} \sigma(x)$ representing the number of occupied sites on the lattice.

In the tables and figures following we provide simulation results from three methods, the conventional MH, the CGMC and the two-level CGMC. For the latest method two approaches were tested, the sampling with correction terms, Figures 6- 7 and Tables 4 - 5 and the approximate CG with potential splitting, Figure 6. Figure 5 shows a graph of the interaction potential $J(\mid x-$ $y \mid)$ and $J_{c}(|x-y|)=J(|x-y|)-\bar{J}(|x-y|)$ appearing in the second step of the two-level method, representing the correction of compressing at the first step of the method. $\bar{J}(|x-y|)=\bar{J}(k, l), x, y \in \Lambda_{N}$ with $x \in C_{k}, y \in C_{l}$ is the compressed potential, used for the coarse space simulations. In all simulations presented in the sequel the range of pure attractive and repulsive forces are $r_{a}=4.47, r_{r}=10$ respectively with repulsion strength $\chi=0.1$. Since the interactions follow an exponential decay, in implementations we use a cut-off range $L$ for the potential $J(r)$ such that $J(r)<10^{-6}$ for $r>L$, which accounts on $(2 L)^{2}$ interactions for each site.

For total coverage $c_{0}=0.9$, that leads to pattern composed by periodic inverted discs, Table 4 gives the average discs diameter and computational time for different values of lattice size $N$, obtained with the two-level CGMC method with the corrections strategy. The results verify that the proposed method provides the expected behaviour of the system for a finite lattice as its size increases, that is features properties stabilize. Features statistics are calculated with the use of edge detection techniques of image processing [32]. In order to demonstrate the effect of the statistical errors we present 


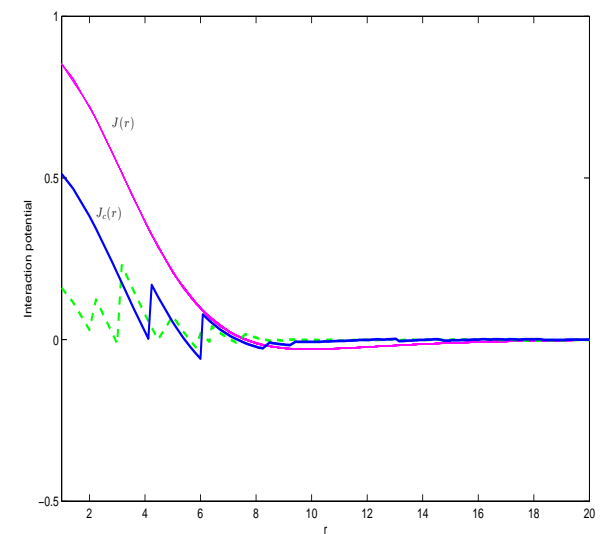

Figure 5: Correcting compressed interactions.

confidence interval estimates. Such intervals are obtained after transforming the simulations data to follow an approximately normal distribution. In simulations for all methods the number of $\mathrm{MC}$ iterations is $5 \times 10^{7}$. For

Table 4: Finite system size behaviour of the two-level CGMC method. Average discs diameter $\langle d\rangle$ and computational time. $c_{0}=0.9, \beta J_{0}=0.6 q=8, L=24$.

\begin{tabular}{ccccc}
\hline Lattice size & Diameter & Std & Confidence Interval & CPU time $(\min )$ \\
\hline $128 \times 128$ & 10.2 & 3.6 & {$[5.7,13.3]$} & 30 \\
$256 \times 256$ & 9.5 & 2.5 & {$[8.7,9.9]$} & 43 \\
$512 \times 512$ & 8.8 & 3.4 & {$[8.5,9.1]$} & 66 \\
$1024 \times 1024$ & 8.6 & 3.6 & {$[8.5,8.7]$} & 110
\end{tabular}

\begin{tabular}{ccccc}
\hline MH method & & & & \\
\hline $512 \times 512$ & 8.27 & 1.2 & {$[8.1,8.3]$} & 805
\end{tabular}

the sake of comparison in Table 4 we present also results obtained with the MH method for a $N=512 \times 512$ lattice. For the same lattice the CGMC method was tested with coarse graining parameter $q=8$ that shows significant deviations, the average diameter being $\langle d\rangle \sim q \times<\bar{d}\rangle=8 \times 7.8$. This proves that we over-coarsened, on the other hand for smaller values of the parameter $Q$ CGMC method provides better approximations, for example when $q=2<\bar{d}>=4.9$ and $<d>\sim 2 \times 4.9$, but the computational time, being 
187 minutes, is still relatively large.

Figures 6 and 7 show the averaged equilibrium conformations for two total coverage values $c_{0}=0.9$ and $c_{0}=0.5$, leading to the formation of inverted discs and stripes respectively, for a $N=256 \times 256$ lattice. Black and white dots indicate occupied and vacant sites respectively. CGMC sampling is fast (i.e. 7.3 minutes for $q=8$ ) but with large deviations from the expected pattern are significant, while the two-level CGMC refines that error providing correct feature scales, of course with the cost of increasing the computational, see Figures $7 \mathrm{~b}$ and $7 \mathrm{c}$. On the other hand there is a substantial reduce on computational time compared to the $\mathrm{MH}$ method, from 365 to 25 minutes. For a smaller coarse graining parameter, $q=4$ in Figure 7d, the CGMC prediction is better albeit at a higher computational cost where again and the coarsening error is improved by the proposed method and microscopic details are available.

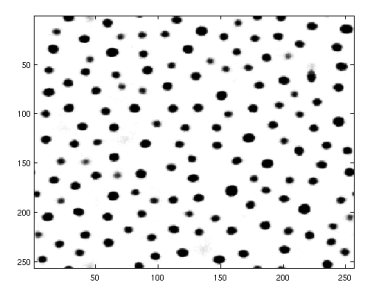

(a) $\mathrm{MH}$

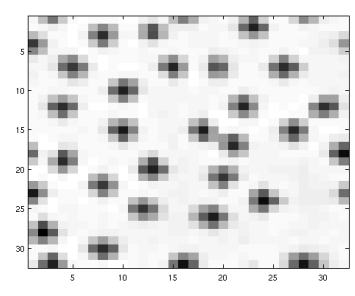

(b) CGMC, $q=8$

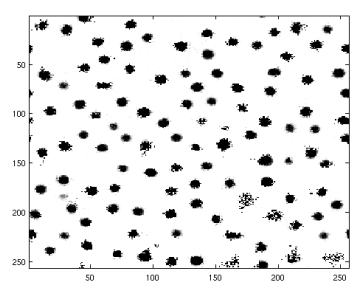

(c) Two-level, $\mathrm{q}=8$

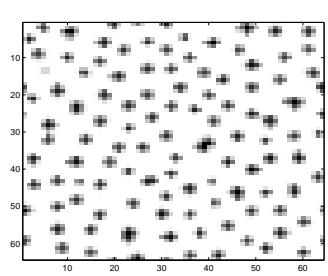

(d) CGMC, $q=4$

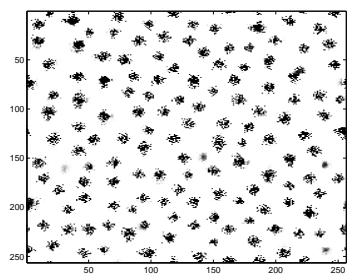

(e) Two-level, $\mathrm{q}=4$

Figure 6: The two-level method with correction is independent of the coarsening parameter. Inverted discs $c_{0}=0.9, \beta J_{0}=0.6, N=256 \times 256$.

Table 5 shows a comparison of the discs statistics and computational time for the conventional and the two-level method for various coarse graining parameters. The two-level method estimates are close to the MH even for high values of the coarse parameter, with a significant reduction of the 


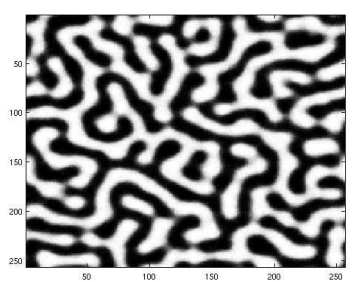

(a) $\mathrm{MH}$

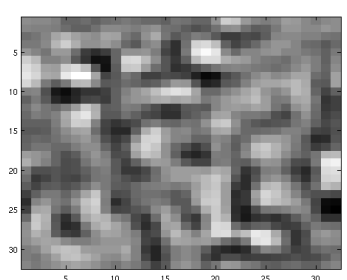

(b) CGMC, $q=8$

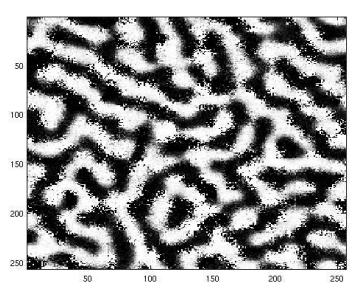

(c) Two-level, $\mathrm{q}=8$

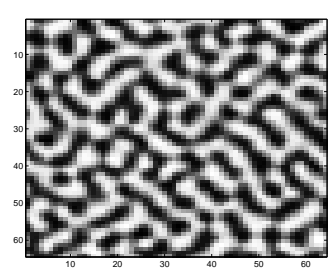

(d) CGMC, $q=4$

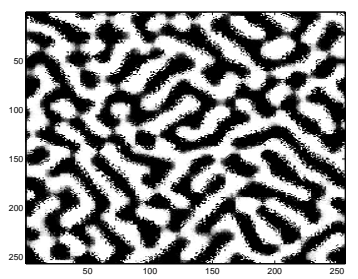

(e) Two-level, $q=4$

Figure 7: The two-level method captures the correct feature scaling even when CGMC is inaccurate. Labyrinths $c_{0}=0.5, \beta J_{0}=0.2, N=256 \times 256$.

computational time.

Table 5: Two-level CGMC with correction, $N=256 \times 256$

\begin{tabular}{cccc}
\hline Method & Diameter $\langle d>$ & Std & CPU time $(\min )$ \\
\hline MH & 8.7 & 1.3 & 252 \\
Two-level $\mathrm{q}=2$ & 8.4 & 1.6 & 66 \\
Two-level $\mathrm{q}=4$ & 7.9 & 4.6 & 22 \\
Two-level $\mathrm{q}=8$ & 8.9 & 3.2 & 23 \\
\hline
\end{tabular}

Figures 8 present results of simulations with the approximate splitting approach where we split the interactions up to range $S$ and neglect the correction terms at the second step, see Section 3.1. This strategy captures the qualitative picture but misses the characteristic length. From the simulations we tested we conclude that this approach is not suggested since the splitting disturbs significuntly the relative strength of attractive and repulsive forces that has an impact on characteristic length scales of the features. 


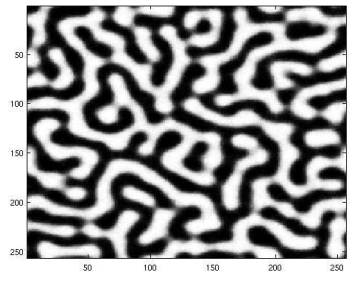

(a) $\mathrm{MH}$

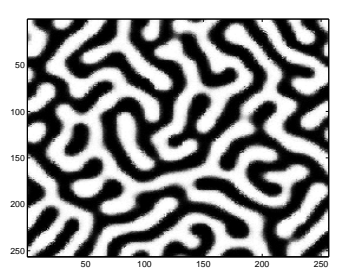

(b) Splitting

Figure 8: Labyrinths. Coupled method with splitting potential, $q=8, s=4$. Splitting captures the qualitative picture but misses the characteristic scale.

\section{Conclusions}

In this work we propose the multilevel CGMC method and study its efficiency theoretically and numerically. The hierarchy of CGMC methods introducing multilevel decompositions are shown to reduce the computational complexity of conventional methods. The reconstructing measures, implemented by an accept/reject method, provide the correct inverse mapping from coarse to fine resolutions refining CGMC sampling. With Theorem 2 we prove that in terms of speed of convergence the two-level CGMC is comparable to a conventional $\mathrm{MH}$, that is controlled by the coarse approximating measure $\bar{\mu}^{(0)}(d \eta)$. In general the method's mixing time cannot be better than a conventional method, but the reduction of computational effort with the error control of the coarse-graining approximation make it an adequate method for studying high dimensional systems. We successfully apply the method in a nano-pattern discovery problem, verifying that the multilevel CGMC method provides the expected behaviour of the system and microscopic information, even when CGMC is inaccurate.

\section{Acknowledgements}

\section{Appendix A. Proofs}

Before studying the proposed method's mathematical properties we need to introduce some definitions and theoretical facts [33]. Let $\left\{X_{n}\right\}$ be a Markov chain on space $\Sigma$ with transition kernel $\mathcal{K}$.

Definition 1. i) A transition kernel $\mathcal{K}$ has the stationary measure $\mu$ if

$$
\int_{\Sigma} \mathcal{K}\left(\sigma, \sigma^{\prime}\right) \mu\left(d \sigma^{\prime}\right)=\mu(\sigma), \text { for all } \sigma \in \Sigma .
$$


ii) $\mathcal{K}$ is called reversible with respect to $\mu$ if

$$
(g, \mathcal{K} f)_{\mu}=(\mathcal{K} g, f)_{\mu}, \quad \text { for all } g, f \in L^{2}(\mu) .
$$

where $(g, f)_{\mu}=\int_{\Sigma} \overline{g(\sigma)} f(\sigma) \mu(d \sigma), \bar{g}$ denoting the complex conjugate of $g$ and $\mathcal{K} g(\sigma)=\int_{\Sigma} \mathcal{K}\left(\sigma, d \sigma^{\prime}\right) g\left(\sigma^{\prime}\right), \forall \sigma \in \Sigma$. A sufficient condition for $\mu$ being a stationary measure of $\mathcal{K}$ that is often easy to check is the detailed balance (DB) condition.

Definition 2. (Detailed Balance) A Markov chain with transition kernel $\mathcal{K}$ satisfies the detailed balance condition if there exists a function $f$ satisfying

$$
\mathcal{K}\left(\sigma, \sigma^{\prime}\right) f(\sigma)=\mathcal{K}\left(\sigma^{\prime}, \sigma\right) f\left(\sigma^{\prime}\right), \text { for all } \sigma, \sigma^{\prime} \in \Sigma .
$$

Here we continue with the proof of Theorem 1 .

Proof. i) Let $\sigma \neq \sigma^{\prime}$, recalling the definition of transition kernel $\mathcal{K}_{C G}\left(\sigma, \sigma^{\prime}\right)$ (18) we have

$$
\begin{aligned}
\mathcal{K}_{C G}\left(\sigma, \sigma^{\prime}\right) \mu(\sigma)= & \alpha_{f}\left(\sigma, \sigma^{\prime}\right) \alpha_{C G}\left(\mathbf{T} \sigma, \mathbf{T} \sigma^{\prime}\right) \mu_{r}\left(\sigma^{\prime} \mid \mathbf{T} \sigma^{\prime}\right) \bar{\rho}\left(\mathbf{T} \sigma, \mathbf{T} \sigma^{\prime}\right) \mu(\sigma) \\
= & \min \left\{1, \frac{\mu\left(\sigma^{\prime}\right) \bar{\mu}^{(0)}(\mathbf{T} \sigma) \mu_{r}(\sigma \mid \mathbf{T} \sigma)}{\mu(\sigma) \bar{\mu}^{(0)}\left(\mathbf{T} \sigma^{\prime}\right) \mu_{r}\left(\sigma^{\prime} \mid \mathbf{T} \sigma^{\prime}\right)}\right\} \times \\
& \min \left\{1, \frac{\bar{\mu}^{(0)}\left(\mathbf{T} \sigma^{\prime}\right) \bar{\rho}\left(\mathbf{T} \sigma^{\prime}, \mathbf{T} \sigma\right)}{\bar{\mu}^{(0)}(\mathbf{T} \sigma) \bar{\rho}\left(\mathbf{T} \sigma, \mathbf{T} \sigma^{\prime}\right)}\right\} \mu_{r}\left(\sigma^{\prime} \mid \mathbf{T} \sigma^{\prime}\right) \bar{\rho}\left(\mathbf{T} \sigma, \mathbf{T} \sigma^{\prime}\right) \mu(\sigma) \\
= & \min \left\{1, \frac{\mu(\sigma) \bar{\mu}^{(0)}\left(\mathbf{T} \sigma^{\prime}\right) \mu_{r}\left(\sigma^{\prime} \mid \mathbf{T} \sigma^{\prime}\right)}{\mu\left(\sigma^{\prime}\right) \bar{\mu}^{(0)}(\mathbf{T} \sigma) \mu_{r}(\sigma \mid \mathbf{T} \sigma)}\right\} \times \\
& \min \left\{1, \frac{\bar{\mu}^{(0)}(\mathbf{T} \sigma) \bar{\rho}\left(\mathbf{T} \sigma, \mathbf{T} \sigma^{\prime}\right)}{\bar{\mu}^{(0)}\left(\mathbf{T} \sigma^{\prime}\right) \bar{\rho}\left(\mathbf{T} \sigma^{\prime}, \mathbf{T} \sigma\right)}\right\} \mu_{r}(\sigma \mid \mathbf{T} \sigma) \bar{\rho}\left(\mathbf{T} \sigma^{\prime}, \mathbf{T} \sigma\right) \mu\left(\sigma^{\prime}\right) \\
= & \mathcal{K}_{C G}\left(\sigma^{\prime}, \sigma\right) \mu\left(\sigma^{\prime}\right) .
\end{aligned}
$$

ii) follows from $i$ ). Detailed balance with $\mu(\sigma)$ is sufficient to guarantee that $\mu(\sigma)$ is the stationary distribution of kernel $\mathcal{K}_{C G}\left(\sigma, \sigma^{\prime}\right)$, (Theorem 6.46 in [33]).

iii) To prove that chain $\left\{Y_{n}\right\}$ is $\mu$-irreducible we need to prove $\mathcal{K}_{C G}(\sigma, A)>0$, for all $\sigma \in E$ and $A$ measurable such that $\mu(A)>0$. We have

$$
\begin{aligned}
\mathcal{K}_{C G}(\sigma, A) & =\int_{A} \mathcal{K}_{C G}\left(\sigma, \sigma^{\prime}\right) d \sigma^{\prime} \geq \int_{A-\{\sigma\}} \mathcal{K}_{C G}\left(\sigma, \sigma^{\prime}\right) d \sigma^{\prime}= \\
& =\int_{A-\{\sigma\}} \alpha_{f}\left(\sigma, \sigma^{\prime}\right) \alpha_{C G}\left(\mathbf{T} \sigma, \mathbf{T} \sigma^{\prime}\right) \mu_{r}\left(\sigma^{\prime} \mid \mathbf{T} \sigma^{\prime}\right) \bar{\rho}\left(\mathbf{T} \sigma, \mathbf{T} \sigma^{\prime}\right) d \sigma^{\prime}
\end{aligned}
$$


From assumptions on $\bar{\rho}\left(\eta, \eta^{\prime}\right)$ and $\mu_{r}(\sigma \mid \eta)$ term $\mu_{r}\left(\sigma^{\prime} \mid \mathbf{T} \sigma^{\prime}\right) \bar{\rho}\left(\mathbf{T} \sigma, \mathbf{T} \sigma^{\prime}\right)$ is positive for all $\sigma, \sigma^{\prime} \in E$. Also since $A \subset E$ and $E \subset \operatorname{supp}\left(\mu_{0}\right), \alpha_{f}\left(\sigma, \sigma^{\prime}\right)$ and $\alpha_{C G}\left(\mathbf{T} \sigma, \mathbf{T} \sigma^{\prime}\right)$ are positive. These ensure that $\mathcal{K}_{C G}(\sigma, A)>0$.

iv) A sufficient condition ensuring that $\left\{Y_{n}\right\}$ is aperiodic is that $\mathcal{K}(\sigma,\{\sigma\})>0$ for some $\sigma \in E$, that means the event $Y_{n+1}=Y_{n}$ happens with positive probability. We have

$$
\mathcal{K}_{C G}(\sigma,\{\sigma\})=1-\int_{\left\{\sigma^{\prime} \neq \sigma\right\}} \alpha_{f}\left(\sigma, \sigma^{\prime}\right) \alpha_{C G}\left(\mathbf{T} \sigma, \mathbf{T} \sigma^{\prime}\right) \mu_{r}\left(\sigma^{\prime} \mid \mathbf{T} \sigma^{\prime}\right) \bar{\rho}\left(\mathbf{T} \sigma, \mathbf{T} \sigma^{\prime}\right) d \sigma^{\prime}
$$

If for all $\sigma \in \Sigma, \mathcal{K}_{C G}(\sigma,\{\sigma\})=0$ then

$$
\int_{\left\{\sigma^{\prime} \neq \sigma\right\}} \alpha_{f}\left(\sigma, \sigma^{\prime}\right) \alpha_{C G}\left(\mathbf{T} \sigma, \mathbf{T} \sigma^{\prime}\right) \mu_{r}\left(\sigma^{\prime} \mid \mathbf{T} \sigma^{\prime}\right) \bar{\rho}\left(\mathbf{T} \sigma, \mathbf{T} \sigma^{\prime}\right) d \sigma^{\prime}=1,
$$

which means that $\alpha_{f}\left(\sigma, \sigma^{\prime}\right)=1$ and $\alpha_{C G}\left(\mathbf{T} \sigma, \mathbf{T} \sigma^{\prime}\right)=1$ for almost all $\sigma \in$ $\left\{\sigma \in E: \bar{\rho}\left(\mathbf{T} \sigma, \mathbf{T} \sigma^{\prime}\right) \mu_{r}\left(\sigma^{\prime} \mid \mathbf{T} \sigma^{\prime}\right)>0\right.$, for some $\left.\sigma^{\prime} \in E\right\}$. This would mean that the reconstructed proposal kernel $\bar{\rho}\left(\mathbf{T} \sigma, \mathbf{T} \sigma^{\prime}\right) \mu_{r}\left(\sigma^{\prime} \mid \mathbf{T} \sigma^{\prime}\right)$ is sampling from the exact target measure $\mu$ which in general is not true. Therefore there exists $\sigma \in E$ such that $\mathcal{K}_{C G}(\sigma,\{\sigma\})=0$.

\section{Appendix B. Benchmark example calculations}

The detailed calculations of the application of Theorem 2 for the benchmark example described in Section 5.1 are provided in detail here.

The conventional MH algorithm is described by a transition kernel proposing a spin-flip at the site $\mathrm{x}$ with the probability $1 / N$, that is $\rho\left(\sigma, \sigma^{\prime}\right)=$ $N^{-1} \sum_{x \in \Lambda_{N}} \delta\left(\sigma^{\prime}-\sigma^{x}\right)$, with acceptance probability

$$
\alpha\left(\sigma, \sigma^{x}\right)=\min \left\{1, e^{(1-2 \sigma(x))\left[K(\sigma(x-1)+\sigma(x+1))+\frac{J}{N} \sum_{y \neq x}^{N} \sigma(y)\right]}\right\}
$$

where, for simplicity, we consider the case of zero external field $h$. In the two-level CGMC algorithm the proposal probability distribution is

$$
\bar{\rho}\left(\eta, \eta^{\prime}\right)=\frac{1}{M} \sum_{k \in \bar{\Lambda}_{M}}\left[\frac{Q-\eta(k)}{Q} \delta\left(\eta^{\prime}-\eta^{k+}\right)+\frac{\eta(k)}{Q} \delta\left(\eta^{\prime}-\eta^{k-}\right)\right]
$$

where $\eta^{k \pm}(l)=\eta(l), l \neq k$ and $\eta^{k \pm}(k)=\eta(k) \pm 1$ and $k \pm$ denotes the adsorption $(+)$ or desorption $(-)$ in the cell $k$. The acceptance probability of the first level is

$$
\alpha_{C G}\left(\eta, \eta^{k \pm}\right)=\min \left\{1, e^{-\Delta_{k \pm} \bar{H}_{l}(\eta)}\right\}
$$


where $\Delta_{k+} \bar{H}_{l}(\eta)=-\frac{J}{N} \sum_{l \in \bar{\Lambda}_{M}} \eta(l)$ and $\Delta_{k-} \bar{H}_{l}(\eta)=-\frac{J}{N}\left[1-\sum_{l \in \bar{\Lambda}_{M}} \eta(l)\right]$. The reconstruction probability distribution is a uniform distribution in each cell $\mu_{r}\left(\sigma^{x} \mid \eta^{k \pm}\right)=\prod_{l \in \bar{\Lambda}_{M}} \mu_{r}^{(l)}\left(\sigma_{C_{l}}^{x} \mid \eta^{k \pm}(l)\right)$. Note that at each MC iteration the change in the new state $\eta^{k}$ happens in cell $k$, thus we need to perform the reconstruction of $\sigma^{x}$ only in the cell $k$, according to

$$
\mu_{r}\left(\sigma_{C_{k}}^{x} \mid \eta^{k}(k)\right)=\frac{1}{Q-\eta(k)} \delta(k-k+)+\frac{1}{\eta(k)} \delta(k-k-) .
$$

According to Algorithm 2, the second level acceptance probability is

$$
\alpha_{f}\left(\sigma, \sigma^{x}\right)=\min \left\{1, e^{-\Delta_{x} H_{s}(\sigma)}\right\}=\min \left\{1, e^{K(1-2 \sigma(x))(\sigma(x-1)+\sigma(x+1))}\right\} .
$$

Term $\mathcal{B}\left(\sigma, \sigma^{\prime}\right)(21)$ is equal to one for all $\sigma, \sigma^{\prime} \in \Sigma_{N}$. Indeed for $\eta^{\prime}=\eta^{k+}$ and $x \in C_{k} \subset \Lambda_{N}$,

$$
\frac{\bar{\rho}\left(\eta, \eta^{k+}\right) \mu_{r}\left(\sigma^{x} \mid \eta^{k+}\right)}{\rho\left(\sigma, \sigma^{x}\right)}=\frac{\frac{1}{M} \frac{Q-\eta(k)}{Q} \frac{1}{Q-\eta(k)}}{\frac{1}{N}}=1 .
$$

Similarly for $\eta^{\prime}=\eta^{k-}$,

$$
\frac{\bar{\rho}\left(\eta, \eta^{k-}\right) \mu_{r}\left(\sigma^{x} \mid \eta^{k-}\right)}{\rho\left(\sigma, \sigma^{x}\right)}=\frac{\frac{1}{M} \frac{\eta(k)}{Q} \frac{1}{\eta(k)}}{\frac{1}{N}}=1
$$

Therefore its upper and lower bounds are $\gamma=\bar{\gamma}=1$. We consider the splitting approach, and use that $\left.H_{N}(\sigma)-\bar{H}_{l} \overline{(\eta}\right)=H_{s}(\sigma)$ and $H_{l}(\sigma)=\bar{H}_{l}(\eta)$. The for any $x \in \Lambda_{N}$ such that $x \in C_{k}, k \in \bar{\Lambda}_{M}$ we have

$$
\mathcal{A}\left(\sigma, \sigma^{x}\right)=\left\{\begin{array}{cl}
1, & \text { if }\left(\sigma, \sigma^{x}\right) \in C_{1} \\
\min \left\{e^{-\beta \Delta_{k} \bar{H}_{l}(\eta)}, e^{\beta \Delta_{k} \bar{H}_{l}(\eta)}\right\}, & \text { if }\left(\sigma, \sigma^{x}\right) \in C_{2} \\
\min \left\{e^{-\beta \Delta_{x} H_{s}(\sigma)}, e^{\beta \Delta_{x} H_{s}(\sigma)}\right\}, & \text { if }\left(\sigma, \sigma^{x}\right) \in C_{3} \\
\min \left\{e^{-\beta \Delta_{x} H_{N}(\sigma)}, e^{\beta \Delta_{x} H_{N}(\sigma)}\right\}, & \text { if }\left(\sigma, \sigma^{x}\right) \in C_{4}
\end{array}\right.
$$

Set $C_{4}=\emptyset$ according to the following argument. Let $\left(\sigma, \sigma^{x}\right) \in C_{4}$ such that $\alpha_{C G}=1, \alpha_{f}=1$ and $\alpha<1$. The first two relations are equivalent to $\Delta_{k} \bar{H}_{l}(\eta) \leq 0$ and $\Delta_{x} H_{s}(\sigma) \leq 0$ that imply $\Delta_{x} H_{N}(\sigma) \leq 0$, since $H_{N}(\sigma)=$ $H_{s}(\sigma)+\bar{H}_{l}(\eta)$, thus $\alpha=1$, a contradiction. Analogous argument holds for the case $\alpha=1, \alpha_{C G}<1, \alpha_{f}<1$ that proves $C_{4}=\emptyset$. 
Let us consider $K \neq 0$. Using the analytic expression of $\Delta_{x} H_{s}(\sigma)$ and $\Delta_{k} \bar{H}_{l}(\eta)$ we have, for $\left(\sigma, \sigma^{x}\right) \in C_{2}$

$$
\begin{aligned}
\min \left\{e^{-\beta \Delta_{k+} \bar{H}_{l}(\eta)}, e^{\beta \Delta_{k+} \bar{H}_{l}(\eta)}\right\} & \geq\left. e^{-\left|\beta \Delta_{k+} \bar{H}_{l}(\eta)\right|}\right|_{\left\{\eta(l)=Q, \forall l \in \bar{\Lambda}_{M}\right\}} \\
& =e^{-\left|\frac{J}{N} N\right|}=e^{-|J|} .
\end{aligned}
$$

Similarly for $\left(\sigma, \sigma^{x}\right) \in C_{3}$

$$
\begin{gathered}
\min \left\{e^{-\beta \Delta_{k-} \bar{H}_{l}(\eta)}, e^{\beta \Delta_{k+} \bar{H}_{l}(\eta)}\right\} \geq e^{-\left|\frac{J}{N}(N-1)\right|}>e^{-|J|} \text { and } \\
\min \left\{e^{-\beta \Delta_{x} \bar{H}_{s}(\sigma)}, e^{\beta \Delta_{x} \bar{H}_{s}(\sigma)}\right\} \geq e^{-2|K|} .
\end{gathered}
$$

Therefore $\inf _{\sigma, x} \mathcal{A}\left(\sigma, \sigma^{x}\right)=\left.\mathcal{A}\left(\sigma, \sigma^{x}\right)\right|_{\left\{\sigma: \sigma(x)=1, \forall x \in \Lambda_{N}\right\}}=\min \left\{e^{-|J|}, e^{-2|K|}\right\}$.

When $K=0$ then $C_{2}=\emptyset$, that can be proved with simple arguments, and for $\left(\sigma, \sigma^{x}\right) \in C_{3} \mathcal{A}\left(\sigma, \sigma^{x}\right)=1$ since $\Delta_{x} H_{s}(\sigma)=0$. Proving that $\mathcal{A}\left(\sigma, \sigma^{x}\right)=1$, for all $x \in \Lambda_{N}$ and $\sigma \in \Sigma_{N}$.

\section{References}

[1] G. Arampatzis, M.A. Katsoulakis, P. Plecháč, M. Taufer, L. Xu, Hierarchical fractional-step approximations and parallel kinetic Monte Carlo algorithms.

[2] S. Are, M.A. Katsoulakis, P. Plecháč, L. Rey-Bellet, Multibody interactions in coarse-graining schemes for extended systems, SIAM J. Sci. Comput. 31 (2008) 987-1015.

[3] D.M. Ceperley, Path integrals in the theory of condensed helium, Rev. Mod. Phys. 67 (1995) 279-355.

[4] A. Chatterjee, D. Vlachos, Multiscale spatial Monte Carlo simulations: Multigriding, computational singular perturbation, and hierarchical stochastic closures, J. Chem. Phys. 124 (2006).

[5] A. Chatterjee, D. Vlachos, Systems tasks in nanotechnology via hierarchical multiscale modeling: Nanopattern formation in heteroepitaxy, Chem. Eng. Sci. 62(18-20) (2007) 4852-4863.

[6] J. Dai, W.D. Seider, T. Sinno, Coarse-grained lattice kinetic Monte Carlo simulation of systems of strongly interacting particles, J. Chem. Phys. 128 (2008) 194705. 
[7] A. DeMassi, E. Presutti, Mathematical Methods for Hydrodynamic Limits, Lecture Notes in Mathematics, Springer, New York Berlin Heidelberg, 1991.

[8] P. Diaconis, L. Saloff-Coste, Logarithmic sobolev inequalities for finite markov chains, Ann. Appl. Prob. 6 (1996) 695-750.

[9] S. Duane, A.D. Kennedy, B.J. Pendleton, D. Roweth, Hybrid Monte Carlo, Physics Letters B 195 (1987) 216 - 222.

[10] Y. Efendiev, T. Hou, W. Luo, Preconditioning Markov chain Monte Carlo simulations using coarse-scale models, SIAM J. Sci. Comput. 28 (2006) 776-803.

[11] N. Goldenfeld, Lectures on Phase Transitions and the Renormalization Group, volume 85, Addison-Wesley, New York, 1992.

[12] V.A. Harmandaris, N.P. Adhikari, N.F.A. van der Vegt, K. Kremer, Hierarchical modeling of polysterene: From atomistic to coarse-grained simulations, Macromolecules 39 (2006) 6708.

[13] W.K. Hastings, Monte Carlo Sampling Methods Using Markov Chains and Their Applications, Biometrika 57 (1970) 97-109.

[14] E. Kalligiannaki, M.A. Katsoulakis, P. Plechac, Multilevel kinetic Coarse Graining Monte Carlo methods for stochastic lattice dynamics, in preparation.

[15] E. Kalligiannaki, M.A. Katsoulakis, P. Plechac, Coupled coarse craining and Markov Chain Monte Carlo for lattice systems, Numerical Analysis and Multiscale Computations, Lect. Notes Comput. Sci. Eng. 82 (2011).

[16] M. Kardar, Crossover to equivalent-neighbor multicritical behavior in arbitrary dimensions, Phys. Rev. B 28 (1983) 244-246.

[17] M. Katsoulakis, A. Majda, D. Vlachos, Coarse-grained stochastic processes for microscopic lattice systems, Proc. Natl. Acad. Sci. 100 (2003) $782-787$.

[18] M.A. Katsoulakis, A.J. Majda, D.G. Vlachos, Coarse-grained stochastic processes and Monte Carlo simulations in lattice systems, J. Comp. Phys. 186 (2003) 250-278. 
[19] M.A. Katsoulakis, P. Plechac, L. Rey-Bellet, Numerical and statistical methods for the coarse-graining of many-particle stochastic systems, J. Sci. Comput. 37 (2008) 43-71.

[20] M.A. Katsoulakis, P. Plechac, L. Rey-Bellet, D.K. Tsagkarogiannis, Coarse-graining schemes and a posteriori error estimates for stochastic lattice systems, ESAIM-Math. Model. Numer. Anal. 41 (2007) 627-660.

[21] M.A. Katsoulakis, P. Plecháč, A. Sopasakis, Error analysis of coarsegraining for stochastic lattice dynamics, SIAM J. Numer. Anal. 44 (2006) 2270-2296.

[22] M.A. Katsoulakis, L. Rey-Bellet, P. Plecháč, D. K.Tsagkarogiannis, Mathematical strategies in the coarse-graining of extensive systems: error quantification and adaptivity, J. Non Newt. Fluid Mech. (2008).

[23] M.A. Katsoulakis, L. Rey-Bellet, P. Plecháč, D.K. Tsagkarogiannis, Coarse-graining schemes for stochastic lattice systems with short and long range interactions, submitted to Math. Comp..

[24] M.A. Katsoulakis, J. Trashorras, Information loss in coarse-graining of stochastic particle dynamics, J. Stat. Phys. 122 (2006) 115-135.

[25] M.A. Katsoulakis, D.G. Vlachos, Coarse-grained stochastic processes and kinetic monte carlo simulators for the diffusion of interacting particles, Journal of Chemical Physics 119 (2003) 9412-27.

[26] K. Kremer, F. Müller-Plathe, Multiscale problems in polymer science: simulation approaches, MRS Bull. (March 2001) 205.

[27] T.M. Liggett, Interacting particle systems, Springer, New York Berlin Heidelberg, 1985.

[28] J.S. Liu, Monte Carlo Strategies in Scientific Computing, SpringerVerlag, New York Berlin Heidelberg, 2001.

[29] Liu, J. and C. Sabatti, Simulated Sintering: Markov Chain Monte Carlo With Spaces of Varying Dimensions, Bayesian Statistics, J.M. Bernardo, J.O. Berger, A. P. Dawid, and A.F.M. Smith (eds) 6 (1998) 402-405. 
[30] N. Metropolis, A.W. Rosenbluth,M.N. Rosenbluth, A.H. Teller,E. Teller, Equation of state calculations by fast computing machines, J. Chem. Phys. 21 (1953) 1087-1092.

[31] R.M. Neal, An Improved Acceptance Procedure for the Hybrid Monte Carlo Algorithm, Journal of Computational Physics 111 (1994) 194 203.

[32] J. Parker, Algorithms for Image Processing and Computer Vision, Wiley, 1996.

[33] C.P. Robert, G. Casella, Monte Carlo Statistical Methods, SpringerVerlag, New York, 2004.

[34] B. Simon, The Statistical Mechanics of Lattice Gases, vol. I, Princeton series in Physics, 1993.

[35] J.M. Taylor, P. Plecháč, Multi-level coarse graining methods for sampling stochastic particle systems. 